\title{
Brazilian Trichoptera Checklist II
}

\author{
Henrique Paprocki ${ }^{\dagger}$, Diogo França ${ }^{\dagger}$ \\ † Pontifícia Universidade Católica de Minas Gerais, Museu de Ciências Naturais, Coleção de Invertebrados. Av. Dom José \\ Gaspar, 290, sala 104, Coração Eucarístico, CEP 30535-901, Belo Horizonte, Minas Gerais, Brazil, Belo Horizonte, Brazil
}

Corresponding author: Henrique Paprocki (paprocki@pucminas.br), Diogo França (diogofransa@pucminas.br) Academic editor: Benjamin Price

Received: 20 Aug 2014 | Accepted: 02 Oct 2014 | Published: 09 Oct 2014

Citation: Paprocki H, França D (2014) Brazilian Trichoptera Checklist II. Biodiversity Data Journal 2: e1557. doi: $\underline{\text { 10.3897/BDJ.2.e1557 }}$

\section{Abstract}

A second assessment of Brazilian Trichoptera species records is presented here. A total of 625 species were recorded for Brazil. This represents an increase of $65.34 \%$ new species recorded during the last decade. The Hydropsychidae (124 spp.), followed by the Hydroptilidae (102 spp.) and Polycentropodidae (97 spp.), are the families with the greatest richness recorded for Brazil. The knowledge on Trichoptera biodiversity in Brazil is geographically unequal. The majority of the species is recorded for the southeastern region.

\section{Keywords}

Biodiversity, aquatic insects, Trichoptera, caddisflies, inventory, checklist, Brazil

\section{Introduction}

Biodiversity data should be made widely available online to better inform conservation of natural resources and advancement of natural sciences. Meta-analysis in molecular biology and others areas is currently a major source for understanding complex biological systems (Emerson et al. 2011, Vos et al. 2014). Checklists of animal and plants species and their distribution are one of the most useful sources of information in conservation, 
biodiversity studies, and environmental licensing. Checklists are used by scientists for registering new species records and descriptions, to predict species distribution, to compare environments, and to monitor environmental impacts. As the climate changes, biodiversity data will be very important to understand the adaptation of biological communities to these changes. Conservationists can better predict impact outcomes and propose better conservation plans when checklists are available (Fonseca and Benson 2003).

The first Brazilian Trichoptera checklist was published by Paprocki et al. 2004 and registered 378 species. At that time, the number of people working on Brazilian caddisfly taxonomy could be counted in one hand and almost all of them resided outside of Brazil (e.g. Blahnik, R.J., Flint, O.S., Holzenthal, R.W.) Currently, the number of Trichoptera taxonomists with a Ph.D working and residing in Brazil has increased, but is still low (e.g. Calor, A.R., Dumas, L.L., Nessimian, J.L., Pes, A.M.O., Santos, A.P.M, Takiya, D.M.). More comprehensive checklists with detailed distribution of species would be even more useful in biodiversity analysis and decision making.

The second checklist of Brazilian Trichoptera is presented here with the addition of a historical account of the growth of knowledge on caddisflies in Brazil. This example can be used to understand how growth of an area of study benefits from cooperation and investment.

\section{Materials and methods}

To update the checklist we consulted the "Plataforma Lattes, base de dados de currículos do Conselho Nacional de Desenvolvimento Científico e Tecnológico (CNPq), Ministério da Ciência, Tecnologia e Inovação - Brazil", available in http://lattes.cnpq.brl, which is a federal database of scientists in Brazil. The researchers searched for the keywords Trichoptera, caddisflies, and caddisfly. Searches were concluded in September, 2014.

The search for compiling the Brazilian Trichoptera Checklist was performed on the following databases. The "Web of Science" was searched for Trichoptera and Brazil. All results from this search were examined. The other databases searched were "The Trichoptera World Checklist”, mantained by Dr. John C. Morse, Clemson University, USA; “ Brazilian Caddisflies: Checklist and Bibliography", mantained by Dr. Jorge L. Nessimian research team, Universidade Federal do Rio de Janeiro, Brazil; and "Index to Organism Names", mantained by Thomson Reuters.

The acronyms used for Brazilian states are as listed: $A C$ (Acre), AL (Alagoas), AM (Amazonas), AP (Amapá), BA (Bahia), CE (Ceará), DF (Distrito Federal), ES (Espírito Santo), GO (Goiás), MA (Maranhão), MG (Minas Gerais), MT (Mato Grosso), MS (Mato Grosso do Sul), PA (Pará), PB (Paraíba), PE (Pernambuco), PI (Piauí), PR (Paraná), RJ (Rio de Janeiro), RN (Rio Grande do Norte), RO (Rondônia), RR (Roraima), RS (Rio Grande do Sul), SC (Santa Catarina), SE (Sergipe), SP (São Paulo), TO (Tocantins). Brazil is divided in political regions as following: North (AC, AM, AP, PA, RO, RR, TO), Northeast 
(AL, BA, CE, MA, PB, PE, PI, RN, SE), Southeast (ES, MG, RJ, SP), Midwest (DF, GO, MS, MT) and South (PR, SC, RS).

State names presented in bold letters indicate a new state record from literature.

\section{Anomalopsychidae}

Genus Contulma Flint, 1969

Notes: Flint Jr 1969a, Holzenthal and Flint Jr 1995

Contulma fluminensis Holzenthal \& Robertson, 2006

Distribution: Rio de Janeiro

Notes: Holzenthal and Robertson 2006

Contulma meloi Holzenthal \& Robertson, 2006

Distribution: Sao Paulo

Notes: Holzenthal and Robertson 2006

Contulma sana Jardim \& Nessimian, 2011

Distribution: Rio de Janeiro

Notes: Jardim and Nessimian 2011

Contulma tijuca Holzenthal \& Flint, 1995

Distribution: Rio de Janeiro

Notes: Holzenthal and Flint Jr 1995

Contulma tripui Holzenthal \& Robertson, 2006

Distribution: Minas Gerais

Notes: Holzenthal and Robertson 2006 


\section{Atriplectididae}

Genus Neoatriplectides Holzenthal, 1997

Notes: Holzenthal 1997

Neoatriplectides desiderata Dumas \& Nessimian, 2008

Distribution: Minas Gerais, Rio de Janeiro, Sao Paulo

Notes: Dumas and Nessimian 2008, Dumas and Nessimian 2012

\section{Calamoceratidae}

Genus Phylloicus Müller, 1880

Notes: Müller 1880, Prather 2003

Phylloicus abdominalis (Ulmer), 1905

Distribution: Bahia, Ceara, Minas Gerais, Parana, Rio de Janeiro, Santa Catarina, Sao Paulo

Notes: Ulmer 1905a, Prather 2003, Huamantico et al. 2005, Quinteiro et al. 2014

Phylloicus amazonas Prather, 2003

Distribution: Amazonas

Notes: Prather 2003

Phylloicus angustior Ulmer, 1905

Distribution: Goias, Minas Gerais, Parana, Rio Grande do Sul, Santa Catarina, Sao Paulo

Notes: Ulmer 1905b, Prather 2003, Calor 2011

Phylloicus auratus Prather, 2003

Distribution: Amazonas

Notes: Prather 2003 
Phylloicus bertioga Prather, 2003

Distribution: Sao Paulo

Notes: Prather 2003

Phylloicus bidigitatus Prather, 2003

Distribution: Bahia, Ceara, Minas Gerais, Rio de Janeiro, Sao Paulo

Notes: Prather 2003, Calor 2011, Quinteiro et al. 2014

Phylloicus bromeliarum Müller, 1880

Distribution: Santa Catarina, Sao Paulo

Notes: Müller 1880, Prather 2003

Phylloicus brevior Banks, 1915

Distribution: Parana, Rondonia

Notes: Banks 1915, Prather 2003

Phylloicus camargoi Quinteiro \& Calor, 2011

Distribution: Sao Paulo

Notes: Quinteiro et al. 2011

Phylloicus dumasi Santos \& Nessimian, 2010

Distribution: Amazonas

Notes: Santos and Nessimian 2010b

Phylloicus elektoros Prather, 2003

Distribution: Amazonas

Notes: Prather 2003

Phylloicus fenestratus Flint, 1974

Distribution: Amazonas, Paraiba, Parana, Rondonia, Roraima

Notes: Flint Jr 1974a, Prather 2003 
Phylloicus flinti Prather, 2003

Distribution: Rondonia

Notes: Prather 2003

Phylloicus major Müller, 1880

Distribution: Rio de Janeiro, Santa Catarina, Sao Paulo

Notes: Müller 1880, Prather 2003

Phylloicus monneorum Dumas \& Nessimian, 2010

Distribution: Bahia, Rio de Janeiro

Notes: Dumas and Nessimian 2010b, Quinteiro et al. 2014

Phylloicus obliquus Navás, 1931

Distribution: Bahia, Ceara, Minas Gerais, Rio de Janeiro, Santa Catarina

Notes: Navás 1931, Prather 2003, Quinteiro et al. 2014

Phylloicus paprockii Prather, 2003

Distribution: Bahia, Minas Gerais

Notes: Prather 2003

Phylloicus plaumanni Flint, 1983

Distribution: Santa Catarina

Notes: Flint Jr 1983a, Prather 2003

Phylloicus quadridigitatus Prather, 2003

Distribution: Sao Paulo

Notes: Prather 2003

Phylloicus yolandae Prather, 2003

Distribution: Parana

Notes: Prather 2003 


\section{Ecnomidae}

Genus Austrotinodes Schmid, 1955

Notes: Schmid 1955, Flint Jr 1973, Flint Jr and Denning 1989

Austrotinodes abrachium Thomson \& Holzenthal, 2010

Distribution: Minas Gerais

Notes: Thomson and Holzenthal 2010

Austrotinodes amazonensis Flint \& Denning, 1989

Distribution: Amazonas

Notes: Flint Jr and Denning 1989

Austrotinodes ariasi Flint \& Denning, 1989

Distribution: Amazonas, Para

Notes: Flint Jr and Denning 1989, Dumas et al. 2010

Austrotinodes belchioris Thomson \& Holzenthal, 2010

Distribution: Minas Gerais

Notes: Thomson and Holzenthal 2010

Austrotinodes bracteatus Flint \& Denning, 1989

Distribution: Sao Paulo

Notes: Flint Jr and Denning 1989

Austrotinodes longispinum Thomson \& Holzenthal, 2010

Distribution: Rio de Janeiro, Sao Paulo

Notes: Thomson and Holzenthal 2010

Austrotinodes paraguayensis Flint, 1983

Distribution: Minas Gerais 
Notes: Flint Jr 1983a

Austrotinodes prolixus Flint \& Denning, 1989

Distribution: Minas Gerais, Rio de Janeiro, Sao Paulo

Notes: Flint Jr and Denning 1989, Dumas et al. 2009, Calor 2011

Austrotinodes taquaralis Thomson \& Holzenthal, 2010

Distribution: Rio de Janeiro, Minas Gerais

Notes: Thomson and Holzenthal 2010

Austrotinodes uruguayensis Angrisano, 1994

Distribution: Parana

Notes: Angrisano 1994, Blahnik et al. 2004

\section{Glossosomatidae}

Genus Canoptila Mosely, 1939

Notes: Mosely 1939

Canoptila bifida Mosely, 1939

Distribution: Santa Catarina

Notes: Mosely 1939

Canoptila williami Robertson \& Holzenthal, 2006

Distribution: Sao Paulo, Parana

Notes: Robertson and Holzenthal 2006

Genus Itauara Müller, 1888

Notes: Müller 1888, Flint Jr et al. 1999a, Robertson and Holzenthal 2011 
Itauara alexanderi Robertson \& Holzenthal, 2011

Distribution: Rio de Janeiro

Notes: Robertson and Holzenthal 2011

Itauara amazonica (Flint), 1971

Distribution: Amazonas

Notes: Flint Jr 1971, Robertson and Holzenthal 2011

Itauara blahniki Robertson \& Holzenthal, 2011

Distribution: Sao Paulo

Notes: Robertson and Holzenthal 2011

Itauara brasiliana (Mosely), 1939

Distribution: Santa Catarina

Notes: Mosely 1939, Robertson and Holzenthal 2011

Itauara charlotta Robertson \& Holzenthal, 2011

Distribution: Minas Gerais

Notes: Robertson and Holzenthal 2011

Itauara emilia Robertson \& Holzenthal, 2011

Distribution: Sao Paulo

Notes: Robertson and Holzenthal 2011

Itauara flinti Robertson \& Holzenthal, 2011

Distribution: Sao Paulo

Notes: Robertson and Holzenthal 2011

Itauara jamesii Robertson \& Holzenthal, 2011

Distribution: Minas Gerais

Notes: Robertson and Holzenthal 2011 
Itauara julia Robertson \& Holzenthal, 2011

Distribution: Rio de Janeiro

Notes: Robertson and Holzenthal 2011

Itauara lucinda Robertson \& Holzenthal, 2011

Distribution: Minas Gerais

Notes: Robertson and Holzenthal 2011

Itauara plaumanni (Flint), 1974

Distribution: Santa Catarina

Notes: Flint Jr 1974b, Robertson and Holzenthal 2011

Itauara rodmani Robertson \& Holzenthal, 2011

Distribution: Minas Gerais

Notes: Robertson and Holzenthal 2011

Itauara simplex Robertson \& Holzenthal, 2011

Distribution: Sao Paulo

Notes: Robertson and Holzenthal 2011

Itauara stella Robertson \& Holzenthal, 2011

Distribution: Sao Paulo

Notes: Robertson and Holzenthal 2011

Itauara tusci Robertson \& Holzenthal, 2011

Distribution: Rio de Janeiro

Notes: Robertson and Holzenthal 2011 
Genus Mortoniella Ulmer, 1906

Notes: Ulmer 1906, Mosely 1937, Flint Jr et al. 1999a, Flint Jr et al. 1999b, Blahnik and Holzenthal 2008

Mortoniella acauda Blahnik \& Holzenthal, 2011

Distribution: Santa Catarina

Notes: Blahnik and Holzenthal 2011

Mortoniella agosta Blahnik \& Holzenthal, 2011

Distribution: Minas Gerais, Rio de Janeiro

Notes: Blahnik and Holzenthal 2011

Mortoniella albolineata Ulmer, 1907

Distribution: Santa Catarina, Sao Paulo

Notes: Ulmer 1907a, Blahnik and Holzenthal 2011

Mortoniella alicula Blahnik \& Holzenthal, 2011

Distribution: Rio de Janeiro

Notes: Blahnik and Holzenthal 2011

Mortoniella asymmetris Blahnik \& Holzenthal, 2011

Distribution: Pernambuco

Notes: Blahnik and Holzenthal 2011, Souza et al. 2013a

Mortoniella bocaina Blahnik \& Holzenthal, 2011

Distribution: Sao Paulo

Notes: Blahnik and Holzenthal 2011

Mortoniella catarinensis (Flint), 1974

Distribution: Santa Catarina

Notes: Flint Jr 1974b, Blahnik and Holzenthal 2011 
Mortoniella crescentis Blahnik \& Holzenthal, 2011

Distribution: Rio de Janeiro

Notes: Blahnik and Holzenthal 2011

Mortoniella dolonis Blahnik \& Holzenthal, 2011

Distribution: Minas Gerais, Sao Paulo

Notes: Blahnik and Holzenthal 2011

Mortoniella froehlichi Blahnik \& Holzenthal, 2011

Distribution: Rio de Janeiro

Notes: Blahnik and Holzenthal 2011

Mortoniella guahybae Blahnik \& Holzenthal, 2011

Distribution: Sao Paulo

Notes: Blahnik and Holzenthal 2011

Mortoniella hystricosa Blahnik \& Holzenthal, 2011

Distribution: Santa Catarina

Notes: Blahnik and Holzenthal 2011

Mortoniella intervales Blahnik \& Holzenthal, 2011

Distribution: Sao Paulo

Notes: Blahnik and Holzenthal 2011

Mortoniella latispina Blahnik \& Holzenthal, 2011

Distribution: Rio de Janeiro

Notes: Blahnik and Holzenthal 2011

Mortoniella longispina Blahnik \& Holzenthal, 2011

Distribution: Santa Catarina

Notes: Blahnik and Holzenthal 2011 
Mortoniella meloi Blahnik \& Holzenthal, 2011

Distribution: Sao Paulo

Notes: Blahnik and Holzenthal 2011

Mortoniella ormina (Mosely), 1939

Distribution: Santa Catarina

Notes: Mosely 1939, Blahnik and Holzenthal 2011

Mortoniella parauna Blahnik \& Holzenthal, 2011

Distribution: Minas Gerais

Notes: Blahnik and Holzenthal 2011

Mortoniella paraunota Blahnik \& Holzenthal, 2011

Distribution: Santa Catarina

Notes: Blahnik and Holzenthal 2011

Mortoniella pumila Blahnik \& Holzenthal, 2011

Distribution: Minas Gerais, Rio de Janeiro

Notes: Blahnik and Holzenthal 2011

Mortoniella pusilla Blahnik \& Holzenthal, 2011

Distribution: Minas Gerais

Notes: Blahnik and Holzenthal 2011

Mortoniella teutona (Mosely), 1939

Distribution: Minas Gerais, Parana, Rio de Janeiro, Santa Catarina, Sao Paulo

Notes: Mosely 1939, Blahnik and Holzenthal 2011, Dumas and Nessimian 2012

Mortoniella tripuiensis Blahnik \& Holzenthal, 2011

Distribution: Minas Gerais

Notes: Blahnik and Holzenthal 2011 
Mortoniella truncata Blahnik \& Holzenthal, 2011

Distribution: Minas Gerais

Notes: Blahnik and Holzenthal 2011

Mortoniella unota (Mosely), 1939

Distribution: Santa Catarina

Notes: Mosely 1939, Blahnik and Holzenthal 2011

Mortoniella uruguaiensis Blahnik \& Holzenthal, 2011

Distribution: Santa Catarina

Notes: Blahnik and Holzenthal 2011

Genus Protoptila Banks, 1904

Notes: Banks 1904, Flint Jr 1971, Flint Jr 1996

Protoptila condylifera Flint, 1971

Distribution: Amazonas

Notes: Flint Jr 1971

Protoptila cora Flint, 1983

Distribution: Minas Gerais

Notes: Flint Jr 1983a, Blahnik et al. 2004

Protoptila disticha Flint, 1971

Distribution: Amazonas, Para

Notes: Flint Jr 1971

Protoptila dubitans Mosely, 1939

Distribution: Santa Catarina

Notes: Mosely 1939 
Protoptila ensifera Flint, 1971

Distribution: Amazonas, Para, Roraima

Notes: Flint Jr 1971, Flint Jr 1991

Protoptila flexispina Flint, 1971

Distribution: Amazonas

Notes: Flint Jr 1971

Protoptila longispinata Santos \& Nessimian, 2009

Distribution: Amazonas, Para

Notes: Santos and Nessimian 2009c

Protoptila macilenta Flint, 1971

Distribution: Para

Notes: Flint Jr 1971

Protoptila mara Flint, 1971

Distribution: Amazonas

Notes: Flint Jr 1971

Protoptila simplex Flint, 1971

Distribution: Amazonas, Para

Notes: Flint Jr 1971

Protoptila ternatia Flint, 1971

Distribution: Amazonas

Notes: Flint Jr 1971

Protoptila tetravittata Flint, 1971

Distribution: Amazonas

Notes: Flint Jr 1971 
Protoptila trispicata Flint, 1971

Distribution: Amazonas

Notes: Flint Jr 1971

Genus Tolhuaca Schmid, 1964

Notes: Schmid 1964

Tolhuaca brasiliensis Robertson \& Holzenthal, 2005

Distribution: Sao Paulo

Notes: Robertson and Holzenthal 2005

\section{Helicopsychidae}

Genus Helicopsyche Siebold, 1856

Notes: Siebold 1856, Mülller 1885

Helicopsyche (Cochliopsyche) amica Johanson, 2003

Distribution: Para

Notes: Johanson 2003

Helicopsyche (Cochliopsyche) amazona Johanson, 2003

Distribution: Amazonas

Notes: Johanson 2003

Helicopsyche (Cochliopsyche) blahniki Johanson, 2003

Distribution: Amazonas

Notes: Johanson 2003

Helicopsyche (Cochliopsyche) brazilia Johanson, 2003

Distribution: Minas Gerais

Notes: Johanson 2003 
Helicopsyche (Cochliopsyche) clara (Ulmer), 1905

Distribution: Amazonas, Minas Gerais, Pernambuco, Santa Catarina, Sao Paulo

Notes: Ulmer 1905a, Flint Jr 1996, Johanson 2003, Blahnik et al. 2004, Souza et al. 2013a

Helicopsyche (Cochliopsyche) lobata Flint, 1983

Distribution: Minas Gerais, Santa Catarina

Notes: Flint Jr 1983a, Blahnik et al. 2004

Helicopsyche (Cochliopsyche) opalescens Flint, 1972

Distribution: Amazonas, Minas Gerais, Mato Grosso, Para, Parana, Rio de Janeiro, Rondonia, Roraima, Santa Catarina, Sao Paulo

Notes: Flint Jr 1972, Flint Jr 1996, Johanson 2003, Blahnik et al. 2004

Helicopsyche (Cochliopsyche) pandeirosa Johanson, 2003

Distribution: Minas Gerais

Notes: Johanson 2003

Helicopsyche (Cochliopsyche) xinguensis Johanson, 2003

Distribution: Amazonas, Para

Notes: Johanson 2003

Helicopsyche (Feropsyche) braziliensis (Swainson), 1840

Distribution: Brazil

Notes: Swainson 1840

Helicopsyche (Feropsyche) cipoensis Johanson \& Malm, 2006

Distribution: Minas Gerais

Notes: Johanson and Malm 2006

Helicopsyche (Feropsyche) flinti Johanson, 1999

Distribution: Santa Catarina 
Notes: Johanson 1999

Helicopsyche (Feropsyche) helicoidella (Vallot), 1855

Distribution: Bahia

Notes: Vallot 1855

Helicopsyche (Feropsyche) monda Flint, 1983

Distribution: Minas Gerais, Parana, Rio de Janeiro, Santa Catarina, Sao Paulo Notes: Flint Jr 1983a, Blahnik et al. 2004, Dumas et al. 2009

Helicopsyche (Feropsyche) muelleri Banks, 1920

Distribution: Santa Catarina

Notes: Banks 1920

Helicopsyche (Feropsyche) paprockii Johanson \& Malm, 2006

Distribution: Minas Gerais

Notes: Johanson and Malm 2006

Helicopsyche (Feropsyche) planorboides Machado, 1957

Distribution: Minas Gerais

Notes: Machado 1957

Helicopsyche (Feropsyche) valligera Flint, 1983

Distribution: Santa Catarina

Notes: Flint Jr 1983a

Helicopsyche (Feropsyche) vergelana Ross, 1956

Distribution: Pernambuco

Notes: Ross 1956a, Souza et al. 2013a 
Helicopsyche (Feropsyche) timbira Silva, Santos \& Nessimian, 2014 Distribution: Rio de Janeiro, Sao Paulo Notes: Silva et al. 2014

\section{Hydrobiosidae}

Genus Atopsyche Banks, 1905

Notes: Banks 1905, Schmid 1989

Atopsyche (Atopsaura) acahuana Schmid, 1989

Distribution: Espirito Santo, Minas Gerais, Rio de Janeiro

Notes: Schmid 1989, Blahnik et al. 2004

Atopsyche (Atopsaura) antisuya Schmid, 1989

Distribution: Minas Gerais

Notes: Schmid 1989

Atopsyche (Atopsaura) apurimac Schmid, 1989

Distribution: Rio de Janeiro

Notes: Schmid 1989

Atopsyche (Atopsaura) blahniki Santos \& Holzenthal, 2012

Distribution: Rio de Janeiro

Notes: Santos and Holzenthal 2012

Atopsyche (Atopsaura) galharada Santos \& Holzenthal, 2012

Distribution: Sao Paulo

Notes: Santos and Holzenthal 2012 
Atopsyche (Atopsaura) hamata Ross \& King, 1952

Distribution: Roraima

Notes: Ross and King 1952

Atopsyche (Atopsaura) hatunpuna Schmid, 1989

Distribution: Minas Gerais, Sao Paulo

Notes: Schmid 1989, Dumas and Nessimian 2012

Atopsyche (Atopsaura) huacachaca Schmid, 1989

Distribution: Rio de Janeiro

Notes: Schmid 1989

Atopsyche (Atopsaura) huamachucu Schmid, 1989

Distribution: Rio de Janeiro

Notes: Schmid 1989

Atopsyche (Atopsaura) huanapu Schmid, 1989

Distribution: Rio de Janeiro, Sao Paulo

Notes: Schmid 1989, Blahnik et al. 2004

Atopsyche (Atopsaura) huarcu Schmid, 1989

Distribution: Minas Gerais, Rio de Janeiro, Sao Paulo

Notes: Schmid 1989, Blahnik et al. 2004

Atopsyche (Atopsaura) longipennis (Ulmer), 1905

Distribution: Santa Catarina

Notes: Ulmer 1905a

Atopsyche (Atopsaura) plancki Marlier, 1964

Distribution: Minas Gerais, Rio de Janeiro, Santa Catarina, Sao Paulo

Notes: Marlier 1964b, Blahnik et al. 2004, Santos and Holzenthal 2012 
Atopsyche (Atopsaura) sanctipauli Flint, 1983

Distribution: Minas Gerais, Parana, Rio de Janeiro, Santa Catarina, Sao Paulo

Notes: Flint Jr 1983a, Blahnik et al. 2004

Atopsyche (Atopsaura) serica Ross, 1953

Distribution: Santa Catarina

Notes: Ross 1953

Atopsyche (Atopsaura) siolii Flint, 1971

Distribution: Amazonas

Notes: Flint Jr 1971

Atopsyche (Atopsaura) usingeri Denning \& Sykora, 1968

Distribution: Rio de Janeiro, Sao Paulo

Notes: Denning and Sykora 1968, Calor 2011

Atopsyche (Atopsaura) zernyi Flint, 1974

Distribution: Espirito Santo, Minas Gerais, Rio deJaneiro, Santa Catarina, Sao Paulo Notes: Flint Jr 1974b, Blahnik et al. 2004, Dumas et al. 2010

Atopsyche (Atopsyche) chirihuana Schmid, 1989

Distribution: Minas Gerais

Notes: Schmid 1989, Blahnik et al. 2004

Atopsyche (Atopsyche) erigia Ross, 1947

Distribution: Minas Gerais, Sao Paulo

Notes: Ross 1947, Blahnik et al. 2004, Calor 2011 
Atopsyche (Atopsyche) parauna Santos \& Holzenthal, 2012

Distribution: Minas Gerais

Notes: Santos and Holzenthal 2012

Atopsyche (Atopsyche) urumarca Schmid, 1989

Distribution: Minas Gerais, Sao Paulo

Notes: Schmid 1989, Santos and Holzenthal 2012

\section{Hydropsychidae}

Genus Blepharopus Kolenati, 1859

Notes: Kolenati 1859

Blepharopus diaphanus Kolenati, 1859

Distribution: Espirito Santo, Minas Gerais, Mato Grosso, Rio de Janeiro, Pernambuco, Parana, Roraima, Santa Catarina, Sao Paulo

Notes: Kolenati 1859, Flint Jr 1991, Marinoni and Almeida 2000, Blahnik et al. 2004, Dumas et al. 2009, Nogueira and Cabette 2011, Barcelos-Silva et al. 2012, Souza et al. 2013a, Costa et al. 2014

\section{Genus Centromacronema Ulmer, 1905}

Notes: Ulmer 1905a

Centromacronema auripenne (Rambur), 1842

Distribution: Minas Gerais, Rio de Janeiro, Santa Catarina, Sao Paulo

Notes: Rambur 1842, Dumas et al. 2009, Dumas and Nessimian 2012

Centromacronema obscurum (Ulmer), 1905

Distribution: Minas Gerais, Santa Catarina, Sao Paulo

Notes: Ulmer 1905a, Blahnik et al. 2004 
Genus Leptonema Guérin-Méneville, 1843

Notes: Guérin-Méneville 1843, Flint et al. 1987

Leptonema agraphum (Kolenati), 1859

Distribution: Rio de Janeiro

Notes: Kolenati 1859

Leptonema amazonense Flint, 1978

Distribution: Amazonas, Mato Grosso, Roraima

Notes: Flint Jr 1978, Ribeiro et al. 2009, Nogueira and Cabette 2011

Leptonema amplifurcatum Dumas \& Nessimian, 2009

Distribution: Rio de Janeiro

Notes: Dumas and Nessimian 2009b

Leptonema aspersum (UImer), 1907

Distribution: Bahia, Mato Grosso do Sul, Mato Grosso

Notes: Ulmer 1907c, Nogueira and Cabette 2011

Leptonema aterrimum Mosely, 1933

Distribution: Para

Notes: Mosely 1933

Leptonema bifurcatodes Flint, 2008

Distribution: Rio de Janeiro

Notes: Flint Jr 2008

Leptonema bifurcatum Flint, McAlpine \& Ross, 1987

Distribution: Espirito Santo, Minas Gerais

Notes: Flint et al. 1987, Blahnik et al. 2004 
Leptonema boraceia Flint, McAlpine \& Ross, 1987

Distribution: Rio de Janeiro, Sao Paulo

Notes: Flint et al. 1987

Leptonema columbianum Ulmer, 1905

Distribution: Amazonas, Bahia, Distrito Federal, Goias, Minas Gerais, Mato Grosso do Sul, Para, Rondonia, Sao Paulo

Notes: Ulmer 1905a

Leptonema crassum Ulmer, 1905

Distribution: Espirito Santo, Goias, Minas Gerais, Mato Grosso, Roraima, Sao Paulo

Notes: Ulmer 1905a

Leptonema eugnathum (Müller), 1921

Distribution: Santa Catarina

Notes: Müller 1921

Leptonema ferelunatum Jardim, Dumas \& Nessimian, 2010

Distribution: Rio de Janeiro

Notes: Jardim et al. 2010

Leptonema lacuniferum Flint, 1978

Distribution: Amazonas

Notes: Flint Jr 1978

Leptonema lineaticorne Flint, 2008

Distribution: Minas Gerais

Notes: Flint Jr 2008

Leptonema lunatum Flint, McAlpine \& Ross, 1987

Distribution: Santa Catarina 
Notes: Flint et al. 1987

Leptonema macacu Flint, 2008

Distribution: Rio de Janeiro

Notes: Flint Jr 2008

Leptonema maculatum Mosely, 1933

Distribution: Amazonas, Mato Grosso, Para

Notes: Mosely 1933, Nogueira and Cabette 2011

Leptonema pallidum Guérin-Meneville, 1843

Distribution: Bahia, Ceara, Distrito Federal, Espirito Santo, Goias, Minas Gerais, Rio de Janeiro, Sao Paulo

Notes: Guérin-Méneville 1843, Costa et al. 2014

Leptonema rostratum Flint, McAlpine \& Ross, 1987

Distribution: Amazonas, Mato Grosso, Para, Roraima

Notes: Schmid 1964, Flint et al. 1987, Costa et al. 2014

Leptonema sancticaroli Flint, McAlpine \& Ross, 1987

Distribution: Amazonas

Notes: Flint et al. 1987, Dumas et al. 2010

Leptonema santosi Jardim, Dumas \& Nessimian, 2010

Distribution: Rio de Janeiro

Notes: Jardim et al. 2010

Leptonema serranum Navás, 1933

Distribution: Sao Paulo

Notes: Flint et al. 1987 
Leptonema serratum Jardim, Dumas \& Nessimian, 2010

Distribution: Rio de Janeiro

Notes: Jardim et al. 2010

Leptonema sparsum (Ulmer), 1905

Distribution: Amazonas, Bahia, Distrito Federal, Goias, Minas Gerais, Mato Grosso, Para, Parana, Rio de Janeiro, Rondonia, Roraima, Santa Catarina, Sao Paulo

Notes: Flint et al. 1987, Flint Jr 1991, Marinoni and Almeida 2000, Blahnik et al. 2004, Costa et al. 2014

Leptonema speciosum (Burmeister), 1839

Distribution: Rio de Janeiro

Notes: Burmeister 1839

Leptonema spinulum Flint, McAlpine \& Ross, 1987

Distribution: Amazonas, Distrito Federal, Mato Grosso, Para

Notes: Flint et al. 1987, Dumas et al. 2010

Leptonema stigmaticum Navás, 1916

Distribution: Rio de Janeiro

Notes: Burmeister 1839

Leptonema tholloni Navás, 1923

Distribution: Rio de Janeiro

Notes: Flint et al. 1987

Leptonema tridens Mosely, 1933

Distribution: Minas Gerais, Parana, Rio de Janeiro, Sao Paulo

Notes: Mosely 1933, Blahnik et al. 2004, 
Leptonema trispicatum Flint, McAlpine \& Ross, 1987

Distribution: Parana, Sao Paulo

Notes: Flint et al. 1987, Blahnik et al. 2004

Leptonema viridianum Navás, 1916

Distribution: Bahia, Ceara, Espirito Santo, Goias, Distrito Federal, Minas Gerais, Para, Paraiba, Pernambuco, Parana, Rio de Janeiro, Sao Paulo

Notes: Mosely 1933, Blahnik et al. 2004, Dumas and Nessimian 2012, Barcelos-Silva et al. 2012, Souza et al. 2013a, Costa et al. 2014

Genus Macronema Pictet, 1836

Notes: Pictet 1836, Flint Jr and Bueno-Soria 1982

Macronema amazonense Flint, 1978

Distribution: Amazonas

Notes: Flint Jr 1978

Macronema argentilineatum Ulmer, 1905

Distribution: Amazonas, Para

Notes: Ulmer 1905a

Macronema bicolor Ulmer, 1905

Distribution: Espirito Santo, Minas Gerais, Santa Catarina, Sao Paulo

Notes: Ulmer 1905a, Dumas et al. 2010, Barcelos-Silva et al. 2012

Macronema burmeisteri Banks, 1924

Distribution: Amazonas

Notes: Banks 1924

Macronema exophtalmum Flint, 1978

Distribution: Amazonas

Notes: Flint Jr 1978 
Macronema fragile Banks, 1915

Distribution: Amazonas

Notes: Banks 1915

Macronema fulvum Ulmer, 1905

Distribution: Rio de Janeiro, Sao Paulo

Notes: Ulmer 1905a, Calor 2011

Macronema hageni Banks, 1924

Distribution: Amazonas, Minas Gerais, Mato Grosso, Para, Roraima

Notes: Banks 1924, Flint Jr 1991, Blahnik et al. 2004, Nogueira and Cabette 2011

Macronema immaculatum Mosely, 1934

Distribution: Parana, Sao Paulo

Notes: Mosely 1934a

Macronema lachlani Banks, 1924

Distribution: Amazonas

Notes: Banks 1924

Macronema lineatum Pictet, 1836

Distribution: Bahia

Notes: Pictet 1836

Macronema muelleri Banks, 1924

Distribution: Amazonas

Notes: Banks 1924

Macronema partitum Navás, 1932

Distribution: Rio de Janeiro

Notes: Navás 1932b 
Macronema parvum Ulmer, 1905

Distribution: Amazonas

Notes: Ulmer 1905a

Macronema pennyi Flint, 1978

Distribution: Amazonas

Notes: Flint Jr 1978

Macronema percitans Walker, 1860

Distribution: Amazonas, Mato Grosso, Para

Notes: Walker 1860

Macronema pertyi Banks, 1924

Distribution: Mato Grosso, Para

Notes: Banks 1924

Macronema rubiginosum Guérin-Meneville, 1843

Distribution: Brazil

Notes: Guérin-Méneville 1843

Genus Macrostemum Kolenati, 1859

Notes: Kolenati 1859, Flint Jr and Bueno-Soria 1982, França et al. 2013

Macrostemum arcuatum (Erichson), 1848

Distribution: Amazonas, Mato Grosso, Para

Notes: Erichson 1848, Nogueira and Cabette 2011, França et al. 2013

Macrostemum brasiliense (Fischer, 1970)

Distribution: Bahia, Espirito Santo, Minas Gerais, Rio de Janeiro, Sao Paulo

Notes: Perty 1833, Fischer 1970, Dumas and Nessimian 2012, França et al. 2013 
Macrostemum braueri (Banks), 1924

Distribution: Amazonas

Notes: Banks 1924, França et al. 2013

Macrostemum bravoi França, Paprocki \& Calor, 2013

Distribution: Bahia, Mato Grosso

Notes: França et al. 2013

Macrostemum digramma (McLachlan), 1871

Distribution: Espirito Santo, Minas Gerais, Rio de Janeiro, Sao Paulo

Notes: McLachlan 1871, Calor 2011, Barcelos-Silva et al. 2012, França et al. 2013

Macrostemum erichsoni (Banks), 1920

Distribution: Acre, Amazonas, Bahia, Espirito Santo, Para

Notes: Banks 1920, Barcelos-Silva et al. 2012, França et al. 2013

Macrostemum hyalinum (Pictet), 1836

Distribution: Acre, Bahia, Ceara, Espirito Santo, Mato Grosso, Para, Paraiba, Pernambuco, Parana, Rio de Janeiro, Sao Paulo

Notes: Pictet 1836, Marinoni and Almeida 2000, Dumas et al. 2009, Calor 2011, Nogueira and Cabette 2011, França et al. 2013

Macrostemum negrense (Flint), 1978

Distribution: Amazonas, Mato Grosso, Para

Notes: Flint Jr 1978, França et al. 2013

Macrostemum nigrum França, Paprocki \& Calor, 2013

Distribution: Bahia

Notes: França et al. 2013

Macrostemum par (Navás), 1930

Distribution: Sao Paulo 
Notes: Navás 1930, França et al. 2013

Macrostemum santaeritae (Ulmer), 1905

Distribution: Acre, Amazonas, Bahia, Mato Grosso, Para

Notes: Ulmer 1905b, Nogueira and Cabette 2011, França et al. 2013

Macrostemum surinamense (Flint), 1974

Distribution: Amazonas

Notes: Flint Jr 1974a, França et al. 2013

Macrostemum ulmeri (Banks), 1913

Distribution: Acre, Amazonas, Minas Gerais, Mato Grosso, Para, Pernambuco, Rondonia, Roraima, Sao Paulo

Notes: Banks 1913, Blahnik et al. 2004, Nogueira and Cabette 2011, França et al. 2013, Souza et al. 2013a

Genus Plectromacronema Ulmer, 1906

Notes: Ulmer 1906, Flint Jr 1967, Flint Jr 1983b

Plectromacronema comptum Ulmer, 1906

Distribution: Amazonas, Para, Roraima

Notes: Ulmer 1906, Flint Jr 1991

Plectromacronema subfuscum (Banks), 1920

Distribution: Santa Catarina

Notes: Banks 1920

Genus Pseudomacronema Ulmer, 1905

Notes: Ulmer 1905a

Pseudomacronema vittatum Ulmer, 1905

Distribution: Amazonas, Para 
Notes: Ulmer 1905a

Genus Smicridea McLachlan, 1871

Notes: McLachlan 1871, Flint Jr 1974a, Flint Jr 1989

Smicridea (Rhyacophylax) abrupta Flint, 1974

Distribution: Amazonas, Mato Grosso, Roraima

Notes: Flint Jr 1974a, Flint Jr 1991, Nogueira and Cabette 2011

Smicridea (Rhyacophylax) appendiculata Flint, 1972

Distribution: Amazonas, Minas Gerais, Mato Grosso, Roraima

Notes: Flint Jr 1972, Blahnik et al. 2004, Albino et al. 2011

Smicridea (Rhyacophylax) araguaiense Albino, Pes \& Hamada, 2011

Distribution: MatoGrosso

Notes: Albino et al. 2011

Smicridea (Rhyacophylax) atrobasis Flint, 1983

Distribution: Santa Catarina

Notes: Flint Jr 1983a

Smicridea (Rhyacophylax) bicornuta Albino, Pes \& Hamada, 2011

Distribution: Mato Grosso

Notes: Albino et al. 2011

Smicridea (Rhyacophylax) bifasciata Albino, Pes \& Hamada, 2011

Distribution: Mato Grosso

Notes: Albino et al. 2011

Smicridea (Rhyacophylax) brasiliana (Ulmer), 1905

Distribution: Santa Catarina 
Notes: Ulmer 1905a

Smicridea (Rhyacophylax) caligata Flint, 1974

Distribution: Amazonas

Notes: Flint Jr 1974a

Smicridea (Rhyacophylax) columbiana (Ulmer), 1905

Distribution: Amazonas, Roraima

Notes: Ulmer 1905a, Flint Jr 1991

Smicridea (Rhyacophylax) coronata Flint, 1980

Distribution: Espirito Santo, Minas Gerais, Mato Grosso, Sao Paulo

Notes: Flint Jr 1980, Albino et al. 2011, Barcelos-Silva et al. 2012

Smicridea (Rhyacophylax) dentifera Flint, 1983

Distribution: Sao Paulo

Notes: Flint Jr 1983a, Blahnik et al. 2004

Smicridea (Rhyacophylax) dentisserrata Albino, Pes \& Hamada, 2011

Distribution: Mato Grosso

Notes: Albino et al. 2011

Smicridea (Rhyacophylax) discalis Flint, 1972

Distribution: Minas Gerais, Parana

Notes: Flint Jr 1972, Marinoni and Almeida 2000, Blahnik et al. 2004

Smicridea (Rhyacophylax) ephippifer Flint, 1978

Distribution: Mato Grosso, Para

Notes: Flint Jr 1978, Nogueira and Cabette 2011 
Smicridea (Rhyacophylax) flinti Albino, Pes \& Hamada, 2011

Distribution: Amazonas, Mato Grosso

Notes: Albino et al. 2011

Smicridea (Rhyacophylax) forcipata Flint, 1983

Distribution: Santa Catarina

Notes: Flint Jr 1983a, Blahnik et al. 2004

Smicridea (Rhyacophylax) froehlichi Almeida \& Flint, 2002

Distribution: Minas Gerais, Rio de Janeiro, Sao Paulo

Notes: Almeida and Flint Jr 2002, Dumas et al. 2010

Smicridea (Rhyacophylax) gladiator Flint, 1978

Distribution: Amazonas

Notes: Flint Jr 1978

Smicridea (Rhyacophylax) helenae Albino, Pes \& Hamada, 2011

Distribution: Amazonas, Mato Grosso, Roraima

Notes: Albino et al. 2011

Smicridea (Rhyacophylax) iguazu Flint, 1983

Distribution: Espirito Santo, Minas Gerais, Parana, Rio de Janeiro, Santa Catarina Notes: Flint Jr 1983a, Marinoni and Almeida 2000, Barcelos-Silva et al. 2012

Smicridea (Rhyacophylax) jundiai Almeida \& Flint, 2002

Distribution: Espirito Santo, Minas Gerais, Parana, Rio de Janeiro, Sao Paulo Notes: Almeida and Flint Jr 2002, Dumas et al. 2010

Smicridea (Rhyacophylax) mangaratiba Almeida \& Flint, 2002

Distribution: Rio de Janeiro

Notes: Almeida and Flint Jr 2002 
Smicridea (Rhyacophylax) marlieri Flint, 1978

Distribution: Amazonas, Para

Notes: Flint Jr 1978

Smicridea (Rhyacophylax) marua Flint, 1978

Distribution: Amazonas

Notes: Flint Jr 1978

Smicridea (Rhyacophylax) mesembrina Navás, 1918

Distribution: Mato Grosso

Notes: Navás 1918, Nogueira and Cabette 2011

Smicridea (Ryachophylax) palmar Sganga, 2005

Distribution: Espirito Santo, Pernambuco

Notes: Barcelos-Silva et al. 2012, Souza et al. 2013a

Smicridea (Rhyacophylax) piraya Flint, 1983

Distribution: Espirito Santo, Minas Gerais, Parana, Santa Catarina, Sao Paulo

Notes: Flint Jr 1983a, Marinoni and Almeida 2000, Blahnik et al. 2004, Barcelos-Silva et al. 2012

Smicridea (Rhyacophylax) pseudolobata Flint, 1978

Distribution: Amazonas, Para

Notes: Flint Jr 1978, Dumas et al. 2010

Smicridea (Rhyacophylax) radula Flint, 1974

Distribution: Espirito Santo, Minas Gerais, Parana, Rio de Janeiro, Santa Catarina, Sao Paulo

Notes: Flint Jr 1974c, Blahnik et al. 2004, Dumas et al. 2009, Barcelos-Silva et al. 2012 
Smicridea (Rhyacophylax) ralphi Almeida \& Flint, 2002

Distribution: Espirito Santo, Parana, Rio de Janeiro, Sao Paulo

Notes: Almeida and Flint Jr 2002

Smicridea (Rhyacophylax) roraimense Albino, Pes \& Hamada, 2011

Distribution: Espirito Santo, Pernambuco, Roraima

Notes: Albino et al. 2011, Barcelos-Silva et al. 2012, Souza et al. 2013a

Smicridea (Rhyacophylax) scutellaris Flint, 1974

Distribution: Amazonas, Minas Gerais, Para, Roraima

Notes: Flint Jr 1974a, Flint Jr 1991, Blahnik et al. 2004

Smicridea (Rhyacophylax) spinulosa Flint, 1972

Distribution: Amazonas, Para, Parana, Santa Catarina, Sao Paulo

Notes: Flint Jr 1972, Marinoni and Almeida 2000, Blahnik et al. 2004

Smicridea (Rhyacophylax) unguiculata Flint, 1983

Distribution: Goias, Minas Gerais, Parana, Santa Catarina, Sao Paulo

Notes: Flint Jr 1983a, Marinoni and Almeida 2000, Blahnik et al. 2004

Smicridea (Rhyacophylax) vermiculata Flint, 1978

Distribution: Minas Gerais, Para, Parana, Santa Catarina, Sao Paulo

Notes: Flint Jr 1978, Marinoni and Almeida 2000, Blahnik et al. 2004, Calor 2011

Smicridea (Rhyacophylax) vilela Flint, 1978

Distribution: Amazonas, Para

Notes: Flint Jr 1978

Smicridea (Rhyacophylax) voluta Flint, 1978

Distribution: Amazonas, Para

Notes: Flint Jr 1978 
Smicridea (Rhyacophylax) weidneri Flint, 1972

Distribution: Parana, Santa Catarina

Notes: Flint Jr 1972, Marinoni and Almeida 2000

Smicridea (Smicridea) aequalis Banks, 1920

Distribution: Para

Notes: Banks 1920

Smicridea (Smicridea) albosignata Ulmer, 1907

Distribution: Minas Gerais, Parana, Rio de Janeiro, Sao Paulo

Notes: Ulmer 1907a, Marinoni and Almeida 2000, Blahnik et al. 2004

Smicridea (Smicridea) bivittata (Hagen), 1861

Distribution: Minas Gerais, Para, Sao Paulo

Notes: Hagen 1861, Blahnik et al. 2004, Calor 2011

Smicridea (Smicridea) gemina Blahnik, 1995

Distribution: Espirito Santo

Notes: Blahnik 2005, Barcelos-Silva et al. 2012

Smicridea (Smicridea) mirnae Almeida \& Flint, 2002

Distribution: Parana

Notes: Almeida and Flint Jr 2002

Smicridea (Smicridea) obliqua Flint, 1974

Distribution: Amazonas

Notes: Flint Jr 1974a

Smicridea (Smicridea) palifera Flint, 1981

Distribution: Espirito Santo, Mato Grosso, Pernambuco, Rio de Janeiro, Roraima 
Notes: Flint Jr 1981, Blahnik et al. 2004, Albino et al. 2011, Nogueira and Cabette 2011, Barcelos-Silva et al. 2012, Souza et al. 2013a

Smicridea (Smicridea) paranensis Flint, 1983

Distribution: Minas Gerais, Parana, Rio de Janeiro, Sao Paulo

Notes: Flint Jr 1983a, Marinoni and Almeida 2000, Blahnik et al. 2004, Calor 2011

Smicridea (Smicridea) reinerti Flint, 1978

Distribution: Para

Notes: Flint Jr 1978

Smicridea (Smicridea) sattleri Denning \& Sykora, 1968

Distribution: Sao Paulo

Notes: Denning and Sykora 1968

Smicridea (Smicridea) sexspinosa Flint, 1978

Distribution: Amazonas

Notes: Flint Jr 1978

Smicridea (Smicridea) truncata Flint, 1974

Distribution: Amazonas, Mato Grosso, Para, Rio de Janeiro

Notes: Flint Jr 1974a, Dumas et al. 2009, Nogueira and Cabette 2011

Genus Synoestropsis Ulmer, 1905

Notes: Ulmer 1905a, Flint Jr et al. 1999b

Synoestropsis furcata Flint, 1974

Distribution: Mato Grosso, Para

Notes: Flint Jr 1974a, Flint Jr 1991, Calor 2008a

Synoestropsis grisoli Navás, 1924

Distribution: Amazonas, Bahia, Minas Gerais, Mato Grosso, Para, Roraima 
Notes: Navás 1924, Flint Jr 1991, Blahnik et al. 2004, Nogueira and Cabette 2011, Costa et al. 2014

Synoestropsis obliqua Ulmer, 1905

Distribution: Rio Grande do Sul

Notes: Ulmer 1905a

Synoestropsis pedicillata Ulmer, 1905

Distribution: Espirito Santo, Minas Gerais, Pernambuco, Santa Catarina, Sao Paulo Notes: Ulmer 1905a, Blahnik et al. 2004, Calor 2011, Barcelos-Silva et al. 2012, Souza et al. 2013a

Synoestropsis punctipennis Ulmer, 1905

Distribution: Amazonas

Notes: Ulmer 1905a

Synoestropsis stictonota Navás, 1932

Distribution: Santa Catarina

Notes: Navás 1932a

\section{Hydroptilidae}

\section{Genus Abtrichia Mosely, 1939}

Notes: Mosely 1939

Abtrichia antennata Mosely, 1939

Distribution: Minas Gerais, Pernambuco, Rio de Janeiro, Santa Catarina, Minas Gerais

Notes: Mosely 1939, Blahnik et al. 2004, Dumas et al. 2010

Abtrichia squamosa Mosely, 1939

Distribution: Minas Gerais, Rio de Janeiro, Santa Catarina 
Notes: Mosely 1939, Blahnik et al. 2004

Genus Acostatrichia Mosely, 1939

Notes: Mosely 1939

Acostatrichia brevipenis Flint, 1974

Distribution: Roraima

Notes: Flint Jr 1974a

Acostatrichia plaumanni Mosely, 1939

Distribution: Santa Catarina

Notes: Mosely 1939

Acostatrichia simulans Mosely, 1939

Distribution: Santa Catarina

Notes: Mosely 1939

Genus Alisotrichia Flint, 1964

Distribution: Rondonia

Notes: Flint Jr 1964

Alisotrichia cacaulandia Harris \& Flint, 2002

Distribution: Rondonia

Notes: Harris and Flint Jr 2002

Alisotrichia holzenthali Santos, 2011

Distribution: Minas Gerais

Notes: Santos 2011

Alisotrichia macae Santos, 2011

Distribution: Rio de Janeiro 
Notes: Santos 2011

Alisotrichia nessimiani Santos, 2011

Distribution: Rio de Janeiro

Notes: Santos 2011

Alisotrichia ubatuba Santos, 2011

Distribution: Sao Paulo

Notes: Santos 2011

Genus Anchitrichia Flint, 1970

Notes: Flint Jr 1970

Anchitrichia duplifurcata Flint, 1983

Distribution: Minas Gerais, Rio de Janeiro

Notes: Flint Jr 1983a, Guahyba 1991, Blahnik et al. 2004

Genus Ascotrichia Flint, 1983

Notes: Flint Jr 1983a

Ascotrichia frontalis Flint, 1983

Distribution: Espirito Santo, Rio de Janeiro

Notes: Flint Jr 1983a

Genus Betrichia Mosely, 1939

Notes: Mosely 1939

Betrichia alibrachia Thomson, 2012

Distribution: Rio de Janeiro

Notes: Thomson 2012 
Betrichia hamulifera Flint, 1983

Distribution: Pernambuco, Sao Paulo, Santa Catarina

Notes: Flint Jr 1983a, Calor 2011, Souza et al. 2013b

Betrichia longistyla Flint, 1983

Distribution: Santa Catarina

Notes: Flint Jr 1983a

Betrichia zilbra Mosely, 1939

Distribution: Santa Catarina

Notes: Mosely 1939

Genus Bredinia Flint, 1968

Notes: Flint Jr 1968

Bredinia espinosa Harris, Holzenthal \& Flint, 2002

Distribution: Mato Grosso, Rondonia

Notes: Harris et al. 2002b

Genus Byrsopteryx Flint, 1981

Notes: Flint Jr 1981

Byrsopteryx abrelata Harris \& Holzenthal, 1994

Distribution: Parana, Rio de Janeiro, Sao Paulo

Notes: Harris and Holzenthal 1994, Blahnik et al. 2004, Santos and Nessimian 2010a

Byrsopteryx carioca Santos \& Nessimian, 2010

Distribution: Riode Janeiro

Notes: Santos and Nessimian 2010a 
Byrsopteryx espinhosa Harris \& Holzenthal, 1994

Distribution: Rio de Janeiro

Notes: Harris and Holzenthal 1994

Genus Ceratotrichia Flint, 1992

\section{Ceratotrichia sp.}

Distribution: Amazonas, Rio Grande do Sul

Notes: Flint Jr 1992, Pes and Hamada 2004, Spies et al. 2006

Genus Costatrichia Mosely, 1937

Notes: Mosely 1937

Costatrichia fluminensis Santos \& Nessimian, 2010

Distribution: Rio de Janeiro

Notes: Santos and Nessimian 2010c

Costatrichia nelsonferreirai Santos \& Nessimian, 2010

Distribution: Para

Notes: Santos and Nessimian 2010c

Costatrichia noite Angrisano, 1995

Distribution: Amazonas

Notes: Angrisano 1994, Santos et al. 2013

Costatrichia ipixuna Santos, Takyia \& Nessimian, 2013

Distribution: Amazonas

Notes: Santos et al. 2013

Genus Dicaminus Müller, 1879

Notes: Müller 1879b 
Dicaminus ladislavii Müller, 1879

Distribution: Santa Catarina

Notes: Müller 1879b

Genus Eutonella Muiller, 1921

Notes: Müller 1921, Flint Jr et al. 1999b

Eutonella peltopsychodes Muiller, 1921

Distribution: Santa Catarina

Notes: Müller 1921

Genus Flintiella Angrisano, 1995

Notes: Angrisano 1995

Flintiella andreae Angrisano, 1995

Distribution: Pernambuco

Notes: Angrisano 1995, Souza et al. 2013b

Flintiella astilla Harris, Flint \& Holzenthal, 2002

Distribution: Bahia, Mato Grosso, Parana, Sao Paulo

Notes: Harris et al. 2002a

Flintiella boraceia Harris, Flint \& Holzenthal, 2002

Distribution: Sao Paulo

Notes: Harris et al. 2002a

Flintiella carajas Santos, Jardim \& Nessimian, 2011

Distribution: Para

Notes: Santos et al. 2011 
Flintiella manauara Santos \& Nessimian, 2009

Distribution: Amazonas

Notes: Santos and Nessimian 2009a

Flintiella pizotensis Harris, Flint \& Holzenthal, 2002

Distribution: Amazonas

Notes: Dumas et al. 2010

Genus Hydroptila Dalman, 1819

Notes: Dalman 1819, Bueno-Soria 1984, Harris and Holzenthal 1999

Hydroptila argentinica Flint, 1983

Distribution: Parana, Rio de Janeiro, Sao Paulo

Notes: Flint Jr 1983a, Blahnik et al. 2004, Dumas et al. 2009

Hydroptila producta Mosely, 1939

Distribution: Rio de Janeiro, Santa Catarina

Notes: Mosely 1939, Dumas et al. 2009

Genus Leucotrichia Mosely, 1934

Notes: Mosely 1934b, Flint Jr 1970

Leucotrichia bicornuta Thomson, 2012

Distribution: Rio de Janeiro

Notes: Thomson 2012

Leucotrichia brasiliana Sattler \& Sykora, 1977

Distribution: Amazonas

Notes: Sattler and Sykora 1977 
Genus Metrichia Ross, 1938

Notes: Ross 1938

Metrichia pernambucana Souza \& Santos, 2013

Distribution: Pernambuco

Notes: Souza et al. $2013 b$

\section{Metrichia sp.}

Distribution: Amazonas, Goias, Rio de Janeiro, Rio Grande do Sul, Sao Paulo

Notes: Pes et al. 2005, Spies et al. 2006, Martins-Silva et al. 2008, Spies and Froehlich 2009, Dumas and Nessimian 2012

Genus Neotrichia Morton, 1905

Notes: Morton 1905

Neotrichia abbreviata Flint, 1983

Distribution: Santa Catarina

Notes: Flint Jr 1983a

Neotrichia bellinii Santos \& Nessimian, 2009

Distribution: Amazonas

Notes: Santos and Nessimian 2009b

Neotrichia browni Harris, 1990

Distribution: Amazonas

Notes: Harris 1990, Santos and Nessimian 2009b

Neotrichia colmillosa Harris, 1990

Distribution: Amazonas

Notes: Harris 1990, Santos and Nessimian 2009b 
Neotrichia didii Santos \& Nessimian, 2009

Distribution: Amazonas

Notes: Santos and Nessimian 2009b

Neotrichia djalmasantosi Santos \& Nessimian, 2009

Distribution: Amazonas

Notes: Santos and Nessimian 2009b

Neotrichia dubitans (Mosely), 1939

Distribution: Rio de Janeiro, Santa Catarina

Notes: Mosely 1939, Dumas et al. 2009

Neotrichia durior Flint, 1983

Distribution: Santa Catarina

Notes: Flint Jr 1983a

Neotrichia feolai Santos \& Nessimian, 2009

Distribution: Amazonas, Pernambuco

Notes: Santos and Nessimian 2009b, Souza et al. 2013b

Neotrichia filifera Flint, 1983

Distribution: Minas Gerais, Pernambuco

Notes: Flint Jr 1983a, Blahnik et al. 2004, Souza et al. 2013b

Neotrichia garrinchai Santos \& Nessimian, 2009

Distribution: Amazonas

Notes: Santos and Nessimian 2009b

Neotrichia gilmari Santos \& Nessimian, 2009

Distribution: Amazonas

Notes: Santos and Nessimian 2009b 
Neotrichia longissima Flint, 1983

Distribution: Santa Catarina

Notes: Flint Jr 1983a

Neotrichia niltonsantosi Santos \& Nessimian, 2009

Distribution: Amazonas

Notes: Santos and Nessimian 2009b

Neotrichia noteuna (Mosely), 1939

Distribution: Santa Catarina

Notes: Mosely 1939

Neotrichia novara (Mosely), 1939

Distribution: Santa Catarina

Notes: Mosely 1939

Neotrichia orlandoi Santos \& Nessimian, 2009

Distribution: Amazonas

Notes: Santos and Nessimian 2009b

Neotrichia ovona (Mosely), 1939

Distribution: Santa Catarina

Notes: Mosely 1939

Neotrichia pelei Santos \& Nessimian, 2009

Distribution: Amazonas

Notes: Santos and Nessimian 2009b

Neotrichia rotundata Flint, 1974

Distribution: Roraima

Notes: Flint Jr 1974a 
Neotrichia sicilicula Flint, 1983

Distribution: Santa Catarina

Notes: Flint Jr 1983a

Neotrichia tertia (Mosely), 1939

Distribution: Santa Catarina

Notes: Mosely 1939

Neotrichia teutonia Flint, 1983

Distribution: Santa Catarina

Notes: Flint Jr 1983a

Neotrichia vavai Santos \& Nessimian, 2009

Distribution: Amazonas

Notes: Santos and Nessimian 2009b

Neotrichia zagalloi Santos \& Nessimian, 2009

Distribution: Amazonas

Notes: Santos and Nessimian 2009b

Neotrichia zitoi Santos \& Nessimian, 2009

Distribution: Amazonas

Notes: Santos and Nessimian 2009b

Genus Nothotrichia Flint, 1967

Notes: Flint Jr 1967, Harris and Armitage 1997

Nothotrichia tupi Holzenthal \& Harris, 2002

Distribution: Brazil

Notes: Holzenthal and Harris 2002 
Genus Ochrotrichia Mosely, 1934

Notes: Mosely 1934b

Ochrotrichia caatinga Souza, Santos \& Takiya, 2014

Distribution: Ceará

Notes: Souza et al. 2014

Ochrotrichia concha Bueno \& Santiago, 1992

Distribution: Amazonas

Notes: Bueno-Soria and Santiago-Fragoso 1992

Ochrotrichia constricta Souza, Santos \& Takiya, 2014

Distribution: Bahia

Notes: Souza et al. 2014

Ochrotrichia igrapiuna Souza, Santos \& Takiya, 2014

Distribution: Bahia

Notes: Souza et al. 2014

Ochrotrichia limeirai Souza, Santos \& Takiya, 2014

Distribution: Ceara

Notes: Souza et al. 2014

Ochrotrichia manuensis Flint \& Bueno-Soria

Distribution: Bahia

Notes: Souza et al. 2014

Ochrotrichia patulosa (Wasmund \& Holzenthal), 2007

Distribution: Ceara, Rio de Janeiro

Notes: Dumas et al. 2009, Oláh and Johanson 2011, Quinteiro et al. 2014 
Ochrotrichia priapo Souza, Santos \& Takiya, 2014

Distribution: Bahia

Notes: Souza et al. 2014

Genus Oxyethira Eaton, 1873

Notes: Eaton 1873

Oxyethira bettyae Thomson \& Holzenthal, 2012

Distribution: Pernambuco

Notes: Thomson and Holzenthal 2012, Souza et al. 2013b

Oxyethira bicornuta Kelley, 1983

Distribution: Amazonas

Notes: Kelley 1983

Oxyethira brasiliensis Kelley, 1983

Distribution: Para

Notes: Kelley 1983

Oxyethira circaverna Kelley, 1983

Distribution: Amazonas

Notes: Kelley 1983, Santos et al. 2009

Oxyethira discaelata Kelley, 1983

Distribution: Amazonas

Notes: Kelley 1983

Oxyethira espinada Holzenthal \& Harris, 1992

Distribution: Minas Gerais

Notes: Harris and Holzenthal 1992, Dumas et al. 2009, Blahnik et al. 2004 
Oxyethira hyalina Müller, 1879

Distribution: Santa Catarina

Notes: Müller 1879b

Oxyethira lagunita Flint, 1983

Distribution: Parana

Notes: Flint Jr 1983a

Oxyethira longipenis Santos, Henriques-Oliveira \& Nessimian, 2009

Distribution: Amazonas

Notes: Santos et al. 2009

Oxyethira longissima Flint, 1974

Distribution: Amazonas

Notes: Flint Jr 1974a, Santos et al. 2009

Oxyethira luanae Santos, Henriques-Oliveira \& Nessimian, 2009

Distribution: Amazonas

Notes: Santos et al. 2009

Oxyethira macrosterna Flint, 1974

Distribution: Amazonas

Notes: Flint Jr 1974a, Santos et al. 2009

Oxyethira merga Kelley, 1983

Distribution: Roraima

Notes: Kelley 1983

Oxyethira parce (Edwards \& Arnold), 1961

Distribution: Minas Gerais

Notes: Edwards and Arnold 1961, Blahnik et al. 2004 
Oxyethira peruviana Harris \& Davenport, 1999

Distribution: Amazonas

Notes: Harris and Davenport 1999, Santos et al. 2009

Oxyethira picita Harris \& Davenport, 1999

Distribution: Amazonas

Notes: Harris and Davenport 1999, Santos et al. 2009

Oxyethira presilla Harris \& Davenport, 1999

Distribution: Amazonas

Notes: Harris and Davenport 1999, Santos et al. 2009

Oxyethira santiagensis Flint, 1982

Distribution: Brazil

Notes: Flint Jr 1982b

Oxyethira sinistra Santos, Henriques-Oliveira \& Nessimian, 2009

Distribution: Amazonas

Notes: Santos et al. 2009

Oxyethira spirogyrae Müller, 1879

Distribution: Santa Catarina

Notes: Müller 1879b

Oxyethira spissa Kelley, 1983

Distribution: Amazonas, Para

Notes: Kelley 1983

Oxyethira tica Holzenthal \& Harris, 1992

Distribution: Amazonas, Minas Gerais, Rio de Janeiro 
Notes: Harris and Holzenthal 1992, Blahnik et al. 2004, Santos et al. 2009, Dumas and Nessimian 2012

Oxyethira zilaba (Mosely), 1939

Distribution: Minas Gerais, Parana, Santa Catarina, Sao Paulo

Notes: Mosely 1939, Blahnik et al. 2004

Genus Peltopsyche Müller, 1879

Notes: Müller 1879b, Flint Jr 1971

Peltopsyche maclachlani Müller, 1879

Distribution: Santa Catarina

Notes: Müller $1879 \mathrm{~b}$

Peltopsyche sieboldii Müller, 1879 sieboldi em Morse

Distribution: Santa Catarina

Notes: Müller 1879b

Genus Rhyacopsyche Müller, 1879

Notes: Müller 1879b

Rhyacopsyche angra Santos, Jardim \& Nessimian, 2011

Distribution: Rio de Janeiro

Notes: Santos et al. 2009

Rhyacopsyche bulbosa Wasmund \& Holzenthal, 2007

Distribution: Minas Gerais, Rio de Janeiro

Notes: Wasmund and Holzenthal 2007

Rhyacopsyche diacantha Santos, Jardim \& Nessimian, 2011

Distribution: Para

Notes: Santos et al. 2011 
Rhyacopsyche dikrosa Wasmund \& Holzenthal, 2007

Distribution: Minas Gerais, Rio de Janeiro, Sao Paulo

Notes: Wasmund and Holzenthal 2007

Rhyacopsyche hagenii Müller, 1879

Distribution: Parana, Rio de Janeiro, Santa Catarina, Sao Paulo

Notes: Müller 1879b, Wasmund and Holzenthal 2007, Dumas et al. 2009

Genus Taraxitrichia Flint \& Harris, 1992

Taraxitrichia sp.

Distribution: Amazonas

Notes: Flint Jr and Harris 1992, Pes and Hamada 2003

Genus Tricholeiochiton Kloet \& Hincks, 1944

Notes: Kloet and Hincks 1944

Tricholeiochiton neotropicalis Flint, 1992

Distribution: Roraima

Notes: Flint Jr 1992

Genus Zumatrichia Mosely, 1937

Notes: Mosely 1937

\section{Zumatrichia sp.}

Distribution: Amazonas

Notes: Mosely 1937, Pes and Hamada 2004 


\section{Leptoceridae}

Genus Achoropsyche Holzenthal, 1984

Notes: Holzenthal 1984

\section{Achoropsyche duodecimpunctata (Navás), 1916}

Distribution: Amazonas, Espirito Santo, Minas Gerais, Mato Grosso, Para, Parana, Rio de Janeiro, Rondonia, Roraima, Santa Catarina, Sao Paulo

Notes: Navás 1916a, Almeida and Marinoni 2000, Blahnik et al. 2004, Dumas et al. 2009, Nogueira and Cabette 2011, Quinteiro et al. 2014

Genus Amazonatolica Holzenthal \& Pes, 2004

Notes: Holzenthal and Pes 2004

Amazonatolica hamadae Holzenthal \& Pes, 2004

Distribution: Amazonas, Bahia, Mato Grosso, Rondonia

Notes: Holzenthal and Pes 2004, Nogueira and Cabette 2011

\section{Genus Atanatolica Mosely, 1936}

Notes: Mosely 1936, Holzenthal 1988b

Atanatolica brasiliana (Brauer), 1865

Distribution: Rio de Janeiro, Sao Paulo

Notes: Brauer 1865, Calor 2011

\section{Atanatolica flinti Holzenthal, 1988}

Distribution: Rio de Janeiro

Notes: Holzenthal 1988b

\section{Genus Grumichella Müller, 1879}

Notes: Müller 1879b, Holzenthal 1988b 
Grumichella aequiunguis Flint, 1983

Distribution: Minas Gerais, Parana, Santa Catarina

Notes: Flint Jr 1983a, Blahnik et al. 2004

Grumichella rostrata Thienemann, 1905

Distribution: Minas Gerais, Rio de Janeiro, Sao Paulo, Santa Catarina

Notes: Thienemann 1905, Holzenthal 1988b

Genus Nectopsyche Müller, 1879

Notes: Müller 1879b

Nectopsyche adusta Flint, 1983

Distribution: Sao Paulo

Notes: Flint Jr 1983a, Calor 2011

Nectopsyche aureovittata Flint, 1983

Distribution: Minas Gerais, Parana, Rio de Janeiro, Santa Catarina, Sao Paulo

Notes: Flint Jr 1983a, Almeida and Marinoni 2000, Blahnik et al. 2004

Nectopsyche acutiloba Flint, 1974

Distribution: Minas Gerais

Notes: Flint Jr 1974a, Blahnik et al. 2004

Nectopsyche bella (Müller), 1921

Distribution: Santa Catarina

Notes: Müller 1921

Nectopsyche bruchi (Navás), 1920

Distribution: Minas Gerais, Parana, Rio de Janeiro

Notes: Navás 1920, Dumas et al. 2009 
Nectopsyche brunneofascia Flint, 1983

Distribution: Sao Paulo, Santa Catarina

Notes: Flint Jr 1983a, Blahnik et al. 2004

Nectopsyche diminuta (Banks), 1920

Distribution: Amazonas, Para, Roraima

Notes: Banks 1920, Flint Jr 1991

Nectopsyche flavofasciata (Ulmer), 1907

Distribution: Minas Gerais, Santa Catarina

Notes: Ulmer 1907a, Blahnik et al. 2004

Nectopsyche fuscomaculata Flint, 1983

Distribution: Minas Gerais, Pernambuco, Parana, Rio de Janeiro, Santa Catarina, Sao Paulo

Notes: Flint Jr 1983a, Almeida and Marinoni 2000, Blahnik et al. 2004, Dumas and Nessimian 2012, Souza et al. 2013a

Nectopsyche gemma (Müller), 1880

Distribution: Santa Catarina

Notes: Müller 1880

Nectopsyche gemmoides Flint, 1981

Distribution: Sao Paulo

Notes: Flint Jr 1981, Calor 2011

Nectopsyche jenseni (Ulmer), 1905

Distribution: Amazonas

Notes: Ulmer 1905b 
Nectopsyche modesta Müller, 1921

Distribution: Santa Catarina

Notes: Müller 1921

Nectopsyche muelleri (Ulmer), 1905

Distribution: Santa Catarina

Notes: Ulmer 1905a

Nectopsyche muhni (Navás), 1916

Distribution: Minas Gerais, Rio de Janeiro, Roraima, Sao Paulo

Notes: Navás 1916b, Flint Jr 1991, Blahnik et al. 2004, Dumas et al. 2009, Calor 2011

Nectopsyche multilineata Flint, 1983

Distribution: Roraima

Notes: Flint Jr 1983a, Flint Jr 1991

Nectopsyche navasi Holzenthal, 1999

Distribution: Santa Catarina

Notes: Flint Jr et al. 1999a

Nectopsyche nigricapilla (Navás), 1922

Distribution: MatoGrosso

Notes: Nogueira and Cabette 2011

Nectopsyche ortizi Holzenthal, 1995

Distribution: Minas Gerais, Para, Parana, Rio de Janeiro,

Notes: Holzenthal 1995, Almeida and Marinoni 2000, Blahnik et al. 2004

Nectopsyche pantosticta Flint, 1983

Distribution: Rio de Janeiro, Rio Grande do Sul, Sao Paulo

Notes: Flint Jr 1983a, Blahnik et al. 2004, Calor 2011 
Nectopsyche punctata (Ulmer), 1905

Distribution: Minas Gerais, Para, Rio de Janeiro, Roraima, Sao Paulo

Notes: Ulmer 1905b, Flint Jr 1991, Blahnik et al. 2004, Dumas et al. 2009

Nectopsyche quatourguttata (Navás), 1922

Distribution: Mato Grosso

Notes: Nogueira and Cabette 2011

Nectopsyche separata (Banks), 1920

Distribution: Minas Gerais, Parana, Rio de Janeiro, Santa Catarina, Sao Paulo

Notes: Banks 1920, Almeida and Marinoni 2000, Blahnik et al. 2004

Nectopsyche splendida (Navás), 1917

Distribution: Bahia, Minas Gerais, Piaui, Parana, Roraima

Notes: Navás 1917, Flint Jr 1991, Almeida and Marinoni 2000, Blahnik et al. 2004, Quinteiro et al. 2014

Genus Neoathripsodes Holzenthal, 1989

Notes: Holzenthal 1989

Neoathripsodes anomalus Holzenthal, 1989

Distribution: Minas Gerais, Rio de Janeiro, Sao Paulo

Notes: Holzenthal 1984, Calor 2011

Genus Notalina Mosely, 1936

Notes: Mosely 1936, Holzenthal 1988b

Notalina (Neonotalina) brasiliana Holzenthal, 1986

Distribution: Minas Gerais

Notes: Holzenthal 1986 
Notalina (Neonotalina) cipo Holzenthal, 1986

Distribution: Minas Gerais

Notes: Holzenthal 1986

Notalina (Neonotalina) froehlichi Calor \& Holzenthal, 2006

Distribution: Minas Gerais

Notes: Calor et al. 2006

Notalina (Neonotalina) goianensis Calor, 2008

Distribution: Goias

Notes: Calor 2008b

Notalina (Neonotalina) hamiltoni Holzenthal, 1986

Distribution: Minas Gerais, Rio de Janeiro, Sao Paulo

Notes: Holzenthal 1986, Dumas and Nessimian 2012

Notalina (Neonotalina) jordanensis Henriques-Oliveira, Spies \& Dumas, 2012

Distribution: Minas Gerais, Sao Paulo

Notes: Henriques-Oliveira et al. 2012

Notalina (Neonotalina) morsei Holzenthal, 1986

Distribution: Minas Gerais, Rio de Janeiro, Sao Paulo

Notes: Holzenthal 1986, Calor et al. 2006, Calor 2011

Notalina (Neonotalina) paulista Calor \& Holzenthal, 2006

Distribution: Rio de Janeiro, Sao Paulo

Notes: Calor et al. 2006

Genus Oecetis McLachlan, 1877

Notes: McLachlan 1877 
Oecetis amazonica (Banks), 1924

Distribution: Amazonas

Notes: Banks 1924

Oecetis angelae Henriques-Oliveria, Dumas \& Nessimian, 2014

Distribution: Mato Grosso do Sul

Notes: Henriques-Oliveira et al. 2014

Oecetis connata Flint, 1974

Distribution: Amazonas, Para

Notes: Flint Jr 1974a, Dumas et al. 2010, Quinteiro et al. 2014

Oecetis danielae Henriques-Oliveria, Dumas \& Nessimian, 2014

Distribution: Amazonas

Notes: Henriques-Oliveira et al. 2014

Oecetis excisa Ulmer, 1907

Distribution: Bahia, Ceara, Pernambuco, Sao Paulo

Notes: Ulmer 1907a, Calor 2011, Souza et al. 2013a, Quinteiro et al. 2014

Oecetis fibra Chen \& Morse, 2012

Distribution: Sao Paulo

Notes: Quinteiro and Calor 2012

Oecetis iara Henriques-Oliveria, Dumas \& Nessimian, 2014

Distribution: Parana

Notes: Henriques-Oliveira et al. 2014

Oecetis inconspicua (Walker), 1852

Distribution: Bahia, Minas Gerais, Paraiba, Parana, Piaui, Sao Paulo

Notes: Walker 1852, Blahnik et al. 2004, Calor 2011, Quinteiro et al. 2014 
Oecetis iguazu Flint, 1983

Distribution: Bahia, Espirito Santo, Minas Gerais, Rio de Janeiro, Santa Catarina, Sao Paulo

Notes: Flint Jr 1983a, Blahnik et al. 2004, Quinteiro et al. 2014

Oecetis paranensis Flint, 1982

Distribution: Bahia, Minas Gerais, Pernambuco

Notes: Flint Jr 1982a, Souza et al. 2013a, Quinteiro et al. 2014

Oecetis punctipennis (Ulmer), 1905

Distribution: Bahia, Minas Gerais, Sao Paulo, Pernambuco, Roraima

Notes: Ulmer 1905b, Flint Jr 1991, Calor 2011, Souza et al. 2013a

Oecetis rafaeli Flint, 1991

Distribution: Roraima

Notes: Flint Jr 1991

Genus Setodes Rambur, 1842

Notes: Rambur 1842

\section{Setodes sp.}

Distribution: Goias

Notes: Barbosa et al. 2011

Genus Triplectides Kolenati, 1859

Notes: Kolenati 1859

Triplectides egleri Sattler, 1963

Distribution: Amazonas, Para

Notes: Sattler 1963 
Triplectides gracilis (Burmeister), 1839

Distribution: Bahia, Espirito Santo, Minas Gerais, Rio de Janeiro, Parana, Santa Catarina, Sao Paulo

Notes: Burmeister 1839, Holzenthal 1988a, Almeida and Marinoni 2000, Quinteiro et al. 2014

Triplectides itatiaia Dumas \& Nessimian, 2010

Distribution: Rio de Janeiro

Notes: Dumas and Nessimian 2010a

Triplectides misionensis Holzenthal, 1988

Distribution: Minas Gerais, Parana, Rio de Janeiro, Santa Catarina, Sao Paulo

Notes: Holzenthal 1988a, Blahnik et al. 2004, Dumas et al. 2010

Triplectides neotropicus Holzenthal, 1988

Distribution: Minas Gerais, Rio de Janeiro, Sao Paulo

Notes: Holzenthal 1988a, Blahnik et al. 2004, Dumas et al. 2009, Calor 2011

Triplectides ultimus Holzenthal, 1988

Distribution: Minas Gerais, Rio de Janeiro

Notes: Holzenthal 1988a, Dumas et al. 2010

\section{Limnephilidae}

Genus Antarctoecia Ulmer, 1907

Notes: Ulmer 1907b

Antarctoecia brasiliensis Huamantinco \& Nessimian, 2003

Distribution: Minas Gerais

Notes: Huamantinco and Nessimian 2003, Huamantinco and Nessimian 2004a 


\section{Odontoceridae}

Genus Anastomoneura Huamantinco \& Nessimian, 2004

Anastomoneura guahybae Huamantinco \& Nessimian, 2004

Distribution: Minas Gerais

Notes: Huamantinco and Nessimian 2004b

Genus Barypenthus Burmeister, 1839

Notes: Burmeister 1839, Flint Jr 1969a, Paprocki and Holzenthal 2002

Barypenthus concolor Burmeister, 1839

Distribution: Bahia, Minas Gerais, Rio de Janeiro, Sao Paulo

Notes: Burmeister 1839, Paprocki and Holzenthal 2002, Quinteiro et al. 2014

Genus Marilia Müller, 1880

Notes: Müller 1880

Marilia aiuruoca Dumas \& Nessimian, 2009

Distribution: Minas Gerais, Rio de Janeiro

Notes: Dumas and Nessimian 2009a

Marilia alata Flint, 1974

Distribution: Amazonas, Pernambuco

Notes: Flint Jr 1974a, Souza et al. 2013a

Marilia albicornis (Burmeister), 1839

Distribution: Sao Paulo

Notes: Burmeister 1839, Calor 2011 
Marilia elongata Martynov, 1912

Distribution: Minas Gerais

Notes: Martynov 1912, Blahnik et al. 2004

Marilia fasciculata Banks, 1913

Distribution: Pernambuco, Rondonia

Notes: Banks 1913, Souza et al. 2013a

Marilia flexuosa Ulmer, 1905

Distribution: Brazil

Notes: Ulmer 1905b

Marilia guaira Flint, 1983

Distribution: Goias, Roraima

Notes: Flint Jr 1983a, Flint Jr 1991

Marilia huamantincoae Dumas \& Nessimian, 2009

Distribution: Rio de Janeiro

Notes: Dumas and Nessimian 2009a

Marilia infundibulum Flint, 1983

Distribution: Santa Catarina

Notes: Flint Jr 1983a

Marilia lateralis Flint, 1983

Distribution: Mato Grosso do Sul

Notes: Flint Jr 1983a

Marilia major Müller, 1880

Distribution: Minas Gerais, Parana, Rio de Janeiro, Santa Catarina

Notes: Müller 1880, Blahnik et al. 2004, Dumas and Nessimian 2009a 
Marilia minor Müller, 1880

Distribution: Minas Gerais, Rio de Janeiro, Santa Catarina

Notes: Müller 1880, Blahnik et al. 2004

Marilia sioli Marlier, 1964

Distribution: Amazonas

Notes: Marlier 1964b

Marilia truncata Flint, 1983

Distribution: Minas Gerais

Notes: Flint Jr 1983a, Blahnik et al. 2004

\section{Philopotamidae}

Genus Alterosa Blahnik, 2005

Notes: Flint Jr 1971, Almeida and Duarte 2003, Blahnik 2005

Alterosa affinis Dumas \& Nessimian, 2013

Distribution: Espirito Santo, Minas Gerais

Notes: Dumas and Nessimian 2013

Alterosa amadoi Dumas, Calor \& Nessimian, 2013

Distribution: Bahia

Notes: Dumas et al. 2013

Alterosa bandeira Dumas \& Nessimian, 2013

Distribution: Espirito Santo, Minas Gerais

Notes: Dumas and Nessimian 2013 
Alterosa beckeri Blahnik, 2005

Distribution: Minas Gerais, Rio de Janeiro

Notes: Blahnik 2005, Dumas et al. 2010

Alterosa bilanceolata Dumas \& Nessimian, 2013

Distribution: Parana

Notes: Dumas and Nessimian 2013

Alterosa bocainae Blahnik, 2005

Distribution: Sao Paulo

Notes: Blahnik 2005

Alterosa boraceiae Blahnik, 2005

Distribution: Sao Paulo

Notes: Blahnik 2005

Alterosa caissara Dumas \& Nessimian, 2013

Distribution: Sao Paulo

Notes: Dumas and Nessimian 2013

Alterosa caparaonensis Blahnik, 2005

Distribution: Espirito Santo, Minas Gerais

Notes: Blahnik 2005, Dumas and Nessimian 2013

Alterosa capixaba Dumas \& Nessimian, 2013

Distribution: Espirito Santo

Notes: Dumas and Nessimian 2013

Alterosa castroalvesi Dumas, Calor \& Nessimian, 2013

Distribution: Bahia

Notes: Dumas et al. 2013 
Alterosa catarinae Dumas \& Nessimian, 2013

Distribution: Santa Catarina

Notes: Dumas and Nessimian 2013

Alterosa caymmii Dumas, Calor \& Nessimian, 2013

Distribution: Bahia

Notes: Dumas et al. 2013

Alterosa escova Blahnik, 2005

Distribution: Rio de Janeiro, Sao Paulo

Notes: Blahnik 2005

Alterosa falcata Blahnik, 2005

Distribution: Minas Gerais, Riode Janeiro, Sao Paulo

Notes: Blahnik 2005

Alterosa fimbriata Blahnik, 2005

Distribution: Rio de Janeiro

Notes: Blahnik 2005

Alterosa flinti Blahnik, 2005

Distribution: Espirito Santo, Minas Gerais, Rio de Janeiro

Notes: Blahnik 2005, Dumas and Nessimian 2013

Alterosa fluminensis Blahnik, 2005

Distribution: Rio de Janeiro

Notes: Blahnik 2005 
Alterosa graciosa Dumas \& Nessimian, 2013

Distribution: Parana

Notes: Dumas and Nessimian 2013

Alterosa guapimirim Blahnik, 2005

Distribution: Rio de Janeiro

Notes: Blahnik 2005

Alterosa holzenthali Blahnik, 2005

Distribution: Santa Catarina

Notes: Blahnik 2005

Alterosa inappendiculata Dumas \& Nessimian, 2013

Distribution: Parana

Notes: Dumas and Nessimian 2013

Alterosa intervales Blahnik, 2005

Distribution: Parana, Sao Paulo

Notes: Blahnik 2005, Dumas and Nessimian 2013

Alterosa itatiaiae Blahnik, 2005

Distribution: Rio de Janeiro

Notes: Blahnik 2005

Alterosa jordaensis Blahnik, 2005

Distribution: Sao Paulo

Notes: Blahnik 2005

Alterosa marinonii (Almeida \& Duarte), 2003

Distribution: Parana, Sao Paulo

Notes: Almeida and Duarte 2003, Blahnik 2005, Dumas and Nessimian 2013 
Alterosa morato Dumas \& Nessimian, 2013

Distribution: Parana

Notes: Dumas and Nessimian 2013

Alterosa nessimiani Jardim \& Dumas, 2012

Distribution: Rio de Janeiro

Notes: Jardim and Dumas 2012

Alterosa orgaosensis Blahnik, 2005

Distribution: Rio de Janeiro

Notes: Blahnik 2005

Alterosa paprockii Blahnik, 2005

Distribution: Minas Gerais

Notes: Blahnik 2005

Alterosa paranaensis Dumas \& Nessimian, 2013

Distribution: Parana

Notes: Dumas and Nessimian 2013

Alterosa ruschii Dumas \& Nessimian, 2013

Distribution: Espirito Santo

Notes: Dumas and Nessimian 2013

Alterosa sanctaeteresae Blahnik, 2005

Distribution: Espirito Santo

Notes: Blahnik 2005

Alterosa sanctipauli (Flint), 1971

Distribution: Sao Paulo

Notes: Flint Jr 1971, Blahnik 2005 
Alterosa schadrackorum Blahnik, 2005

Distribution: Santa Catarina

Notes: Blahnik 2005

Alterosa spiesae Dumas \& Nessimian, 2013

Distribution: Sao Paulo

Notes: Dumas and Nessimian 2013

Alterosa tripuiensis Blahnik, 2005

Distribution: Minas Gerais

Notes: Blahnik 2005

Alterosa truncata Blahnik, 2005

Distribution: Espirito Santo, Minas Gerais, Rio de Janeiro, Sao Paulo

Notes: Blahnik 2005, Dumas and Nessimian 2012, Barcelos-Silva et al. 2012

Genus Chimarra Stephens, 1829

Notes: Stephens 1829, Flint Jr 1998, Blahnik 1998

Chimarra (Chimarra) adamsae Blahnik, 1998

Distribution: Minas Gerais, Para, Parana, Sao Paulo

Notes: Blahnik 1998, Blahnik et al. 2004

Chimarra (Chimarra) calori Blahnik \& Holzenthal, 2012

Distribution: Minas Gerais, Sao Paulo

Notes: Blahnik and Holzenthal 2012

Chimarra (Chimarra) uara Flint, 1971

Distribution: Amazonas, Distrito Federal, Minas Gerais, Para, Pernambuco, Rondonia, Santa Catarina

Notes: Flint Jr 1971, Blahnik et al. 2004, Dumas et al. 2010, Souza et al. 2013a 
Chimarra (Chimarrita) akantha Blahnik, 1997

Distribution: Amazonas

Notes: Blahnik 1997

Chimarra (Chimarrita) camella Blahnik, 1997

Distribution: Minas Gerais, Rio de Janeiro, Sao Paulo

Notes: Blahnik 1997, Blahnik et al. 2004

Chimarra (Chimarrita) camura Blahnik, 1997

Distribution: Rio de Janeiro, Sao Paulo

Notes: Blahnik 1997, Blahnik et al. 2004

Chimarra (Chimarrita) chela Blahnik, 1997

Distribution: Amazonas

Notes: Blahnik 1997, Santos and Nessimian 2009d

Chimarra (Chimarrita) curvipenis Blahnik \& Holzenthal, 2012

Distribution: Minas Gerais

Notes: Blahnik and Holzenthal 2012

Chimarra (Chimarrita) heligma Blahnik, 1997

Distribution: Minas Gerais

Notes: Blahnik 1997

Chimarra (Chimarrita) kontilos Blahnik, 1997

Distribution: Espirito Santo, Minas Gerais, Rio de Janeiro, Sao Paulo

Notes: Blahnik 1997, Blahnik et al. 2004

Chimarra (Chimarrita) latiforceps Blahnik \& Holzenthal, 2012

Distribution: Minas Gerais, Sao Paulo

Notes: Blahnik and Holzenthal 2008 
Chimarra (Chimarrita) majuscula Blahnik, 1997

Distribution: Rio de Janeiro, Sao Paulo

Notes: Blahnik 1997, Blahnik et al. 2004

Chimarra (Chimarrita) simpliciforma Flint, 1971

Distribution: Amazonas

Notes: Flint Jr 1971

Chimarra (Chimarrita) tortuosa Blahnik, 1997

Distribution: Amazonas

Notes: Blahnik 1997

Chimarra (Chimarrita) xingu Blahnik, 1997

Distribution: Para

Notes: Blahnik 1997

Chimarra (Curgia) aurivittata Flint, 1971

Distribution: Amazonas, Rondonia

Notes: Flint Jr 1971, Flint Jr 1998

Chimarra (Curgia) beckeri Flint, 1998

Distribution: Espirito Santo, Minas Gerais, Rio de Janeiro, Sao Paulo Notes: Flint Jr 1998, Dumas et al. 2010, Barcelos-Silva et al. 2012

Chimarra (Curgia) boraceia Flint, 1998

Distribution: Sao Paulo

Notes: Flint Jr 1998

Chimarra (Curgia) brasiliana (Ulmer), 1905

Distribution: Parana, Santa Catarina

Notes: Ulmer 1905a, Almeida and Marinoni 2001 
Chimarra (Curgia) burmeisteri Flint, 1998

Distribution: Rio de Janeiro

Notes: Flint Jr 1998

Chimarra (Curgia) camposae Flint, 1998

Distribution: Minas Gerais

Notes: Flint Jr 1998

Chimarra (Curgia) centrispina Flint, 1998

Distribution: Minas Gerais

Notes: Flint Jr 1998

Chimarra (Curgia) cipoensis Flint, 1998

Distribution: Minas Gerais

Notes: Flint Jr 1998

Chimarra (Curgia) conica Flint, 1983

Distribution: Espirito Santo, Ceara, Goias, Minas Gerais, Mato Grosso, Rio de Janeiro, Rondonia, Santa Catarina

Notes: Flint Jr 1983a, Flint Jr 1998, Blahnik et al. 2004, Barcelos-Silva et al. 2012

Chimarra (Curgia) cultellata Flint, 1983

Distribution: Distrito Federal, Minas Gerais, Para, Rio de Janeiro, Rondonia, Santa Catarina

Notes: Flint Jr 1983a, Flint Jr 1998, Dumas et al. 2009, Dumas et al. 2010

Chimarra (Curgia) donamariae Denning \& Sykora, 1968

Distribution: Para

Notes: Denning and Sykora 1968 
Chimarra (Curgia) fittkaui Flint, 1971

Distribution: Amazonas

Notes: Flint Jr 1971

Chimarra (Curgia) froehlichi Flint, 1998

Distribution: Espirito Santo, Minas Gerais, Rio de Janeiro, Sao Paulo

Notes: Flint Jr 1998, Blahnik et al. 2004

Chimarra (Curgia) hyoeides Flint, 1983

Distribution: Espirito Santo, Para, Pernambuco, Santa Catarina, Sao Paulo

Notes: Flint Jr 1983a, Flint Jr 1998, Barcelos-Silva et al. 2012, Souza et al. 2013a

Chimarra (Curgia) jugescens Flint, 1998

Distribution: Amazonas

Notes: Flint Jr 1998, Santos and Nessimian 2009d

Chimarra (Curgia) medioloba Flint, 1971

Distribution: Amazonas

Notes: Flint Jr 1971

Chimarra (Curgia) morio Burmeister, 1839

Distribution: Bahia, Parana, Rio de Janeiro, Santa Catarina, Sao Paulo

Notes: Burmeister 1839, Flint Jr 1998

Chimarra (Curgia) parana Flint, 1972

Distribution: Espirito Santo, Distrito Federal, Goias, Minas Gerais, Pernambuco, Rio deJaneiro, Santa Catarina, Sao Paulo

Notes: Flint Jr 1972, Flint Jr 1998, Barcelos-Silva et al. 2012, Souza et al. 2013a

Chimarra (Curgia) paucispina Santos \& Nessimian, 2009

Distribution: Amazonas 
Notes: Santos and Nessimian 2009d

Chimarra (Curgia) petersorum Flint, 1998

Distribution: Parana

Notes: Flint Jr 1998

Chimarra (Curgia) petricola Flint, 1998

Distribution: Espirito Santo, Rio de Janeiro

Notes: Flint Jr 1998, Barcelos-Silva et al. 2012

Chimarra (Curgia) plaumanni Flint, 1983

Distribution: Espirito Santo, Santa Catarina

Notes: Flint Jr 1983a, Barcelos-Silva et al. 2012

Chimarra (Curgia) quaternaria Flint, 1971

Distribution: Amazonas

Notes: Flint Jr 1971

Chimarra (Curgia) scopuloides Flint, 1974

Distribution: Goias, Para, Rondonia, Roraima, Santa Catarina

Notes: Flint Jr 1974a, Flint Jr 1998

Chimarra (Curgia) teresae Flint, 1998

Distribution: Espirito Santo, Minas Gerais, Rio de Janeiro, Santa Catarina, Sao Paulo

Notes: Flint Jr 1998, Blahnik et al. 2004, Dumas et al. 2009, Barcelos-Silva et al. 2012

Chimarra (Curgia) tucuna Flint, 1998

Distribution: Amazonas

Notes: Flint Jr 1998 
Chimarra (Curgia) ypsilon Flint, 1983

Distribution: Parana, Rio de Janeiro, Santa Catarina

Notes: Flint Jr 1983a, Almeida and Marinoni 2001

Chimarra (Otarrha) diaksis Flint, 1971

Distribution: Amazonas

Notes: Flint Jr 1971

Chimarra (Otarrha) odonta Blahnik, 2002

Distribution: Espirito Santo, Minas Gerais, Rio de Janeiro, Sao Paulo

Notes: Blahnik 2002, Dumas et al. 2010, Barcelos-Silva et al. 2012

Chimarra (undetermined) usitatissima Flint, 1971

Distribution: Amazonas, Minas Gerais, Para, Rondonia

Notes: Flint Jr 1971, Flint Jr 1974a, Blahnik 1997

Genus Dolophilodes Ulmer, 1909

Notes: Ulmer 1909

Dolophilodes sp.

Distribution: Goias

Notes: Martins-Silva et al. 2008

Genus Wormaldia McLachlan, 1865

Wormaldia planae Ross \& King, 1956

Distribution: Brazil

Notes: Ross 1956a, Flint Jr et al. 1999b 


\section{Polycentropodidae}

Genus Cernotina Ross, 1938

Notes: Ross 1938, Flint Jr 1971

Cernotina abbreviata Flint, 1971

Distribution: Para

Notes: Flint Jr 1971

Cernotina acalyptera Flint, 1971

Distribution: Amazonas

Notes: Flint Jr 1971

Cernotina anhanguera Camargos, Barcelos-Silva \& Pes, 2013

Distribution: Goias

Notes: Barcelos-Silva et al. 2013

Cernotina antonina Holzenthal \& Almeida, 2003

Distribution: Espirito Santo, Minas Gerais, Pernambuco, Parana

Notes: Holzenthal and Almeida 2003, Barcelos-Silva et al. 2013, Souza et al. 2013a

Cernotina aruma Santos \& Nessimian, 2008

Distribution: Amazonas

Notes: Santos and Nessimian 2008

Cernotina attenuata Flint, 1971

Distribution: Amazonas

Notes: Flint Jr 1971 
Cernotina bibrachiata Flint, 1971

Distribution: Amazonas

Notes: Flint Jr 1971

Cernotina bispicata Camargos, Barcelos-Silva \& Pes, 2013

Distribution: Goias

Notes: Barcelos-Silva et al. 2013

Cernotina cacha Flint, 1971

Distribution: Amazonas, Minas Gerais

Notes: Flint Jr 1971, Blahnik et al. 2004

Cernotina cingulata Flint, 1971

Distribution: Amazonas

Notes: Flint Jr 1971

Cernotina compressa Flint, 1971

Distribution: Amazonas

Notes: Flint Jr 1971

Cernotina cygnea Flint, 1971

Distribution: Amazonas

Notes: Flint Jr 1971

Cernotina cystophora Flint, 1971

Distribution: Amazonas

Notes: Flint Jr 1971

Cernotina declinata Flint, 1971

Distribution: Para

Notes: Flint Jr 1971 
Cernotina decumbens Flint, 1971

Distribution: Amazonas

Notes: Flint Jr 1971

Cernotina ecotura Sykora, 1998

Distribution: Roraima

Notes: Sykora 1998

Cernotina encrypta Flint, 1971

Distribution: Amazonas

Notes: Flint Jr 1971

Cernotina falcata Camargos, Barcelos-Silva \& Pes, 2013

Distribution: Goias

Notes: Barcelos-Silva et al. 2013

Cernotina filiformis Flint, 1971

Distribution: Amazonas

Notes: Flint Jr 1971

Cernotina flexuosa Santos \& Nessimian, 2008

Distribution: Amazonas

Notes: Santos and Nessimian 2008

Cernotina lanceolata Barcelos-Silva, Camargos \& Pes, 2013

Distribution: Espirito Santo

Notes: Barcelos-Silva et al. 2012, Barcelos-Silva et al. 2013

Cernotina lazzarii Holzenthal \& Almeida, 2003

Distribution: Parana

Notes: Holzenthal and Almeida 2003 
Cernotina lobisomem Santos \& Nessimian, 2008

Distribution: Amazonas

Notes: Santos and Nessimian 2008

Cernotina longispina Barcelos-Silva, Camargos \& Pes, 2013

Distribution: Espirito Santo

Notes: Barcelos-Silva et al. 2012, Barcelos-Silva et al. 2013

Cernotina obliqua Flint, 1971

Distribution: Amazonas

Notes: Flint Jr 1971

Cernotina odonta Santos \& Nessimian, 2008

Distribution: Amazonas

Notes: Santos and Nessimian 2008

Cernotina perpendicularis Flint, 1971

Distribution: Amazonas, Minas Gerais, Para

Notes: Flint Jr 1971, Blahnik et al. 2004

Cernotina pesae Santos \& Nessimian, 2008

Distribution: Amazonas

Notes: Santos and Nessimian 2008

Cernotina puri Dumas \& Nessimian, 2011

Distribution: Rio de Janeiro

Notes: Dumas and Nessimian 2011

Cernotina sexspinosa Flint, 1983

Distribution: Santa Catarina

Notes: Flint Jr 1983a 
Cernotina sinuosa Barcelos-Silva, Camargos \& Pes, 2013

Distribution: Espirito Santo

Notes: Barcelos-Silva et al. 2012, Barcelos-Silva et al. 2013

Cernotina spinigera Flint, 1971

Distribution: Goias, Para, Roraima

Notes: Flint Jr 1971, Barcelos-Silva et al. 2013

Cernotina spinosior Flint, 1991

Distribution: Roraima

Notes: Flint Jr 1991

Cernotina subapicalis Flint, 1971

Distribution: Amazonas

Notes: Flint Jr 1971

Cernotina trispina Flint, 1971

Distribution: Amazonas

Notes: Flint Jr 1971

Cernotina uara Flint, 1971

Distribution: Amazonas

Notes: Flint Jr 1971

Cernotina unguiculata Flint, 1971

Distribution: Para

Notes: Flint Jr 1971

Cernotina verticalis Flint, 1971

Distribution: Amazonas

Notes: Flint Jr 1971 
Genus Cyrnellus Banks, 1913

Notes: Banks 1913, Flint Jr 1971

Cyrnellus arotron Flint, 1971

Distribution: Amazonas, Mato Grosso do Sul, Para

Notes: Flint Jr 1971, Dumas et al. 2010

\section{Cyrnellus bifidus Flint, 1971}

Distribution: Amazonas, Mato Grosso do Sul

Notes: Flint Jr 1971, Dumas et al. 2010

Cyrnellus collaris Flint, 1971

Distribution: Amazonas

Notes: Flint Jr 1971

Cyrnellus fraternus (Banks), 1905

Distribution: Amazonas, Bahia, Espirito Santo, Minas Gerais, Mato Grosso, Mato Grosso do Sul, Para, Parana, Rio de Janeiro, Santa Catarina

Notes: Banks 1905, Blahnik et al. 2004, Dumas et al. 2009, Dumas et al. 2010, Nogueira and Cabette 2011

Cyrnellus mammillatus Flint, 1971

Distribution: Amazonas, Minas Gerais, Mato Grosso do Sul, Para, Pernambuco, Parana, Rio de Janeiro, Sao Paulo

Notes: Flint Jr 1971, Blahnik et al. 2004, Dumas et al. 2010, Souza et al. 2013a

Cyrnellus risi (Ulmer), 1907

Distribution: Amazonas, Espirito Santo, Minas Gerais, Para

Notes: Ulmer 1907a, Blahnik et al. 2004, Barcelos-Silva et al. 2012 
Cyrnellus ulmeri Flint, 1971

Distribution: Amazonas, Para

Notes: Flint Jr 1971

Genus Nyctiophylax Brauer, 1865

Notes: Brauer 1865, Neboiss 1993

Nyctiophylax neotropicalis Flint, 1971

Distribution: Amazonas, Espirito Santo, Minas Gerais, Para, Pernambuco, Parana, Rio de Janeiro

Notes: Flint Jr 1971, Blahnik et al. 2004, Dumas et al. 2010, Barcelos-Silva et al. 2012, Souza et al. 2013a

Genus Polycentropus Curtis, 1835

Polycentropus acinaciformis Hamilton \& Holzenthal, 2011

Distribution: Minas Gerais

Notes: Hamilton and Holzenthal 2011

Polycentropus amphirhamphus Hamilton \& Holzenthal, 2011

Distribution: Riode Janeiro, Santa Catarina, Sao Paulo

Notes: Hamilton and Holzenthal 2011

Polycentropus ancistrus Hamilton \& Holzenthal, 2011

Distribution: Sao Paulo

Notes: Hamilton and Holzenthal 2011

Polycentropus boraceia Hamilton \& Holzenthal, 2011

Distribution: Sao Paulo

Notes: Hamilton and Holzenthal 2011 
Polycentropus caaete Hamilton \& Holzenthal, 2011

Distribution: Parana, Santa Catarina, Sao Paulo

Notes: Hamilton and Holzenthal 2011

Polycentropus cachoeira Hamilton \& Holzenthal, 2011

Distribution: Santa Catarina

Notes: Hamilton and Holzenthal 2011

Polycentropus carioca Hamilton \& Holzenthal, 2011

Distribution: Rio de Janeiro

Notes: Hamilton and Holzenthal 2011

Polycentropus carolae Hamilton \& Holzenthal, 2011

Distribution: Rio de Janeiro

Notes: Hamilton and Holzenthal 2011

Polycentropus cipoensis Hamilton \& Holzenthal, 2011

Distribution: Minas Gerais, Sao Paulo

Notes: Hamilton and Holzenthal 2011

Polycentropus cheliceratus Hamilton \& Holzenthal, 2011

Distribution: Rio de Janeiro

Notes: Hamilton and Holzenthal 2011

Polycentropus fluminensis Hamilton \& Holzenthal, 2011

Distribution: Espirito Santo, Minas Gerais, Rio de Janeiro

Notes: Hamilton and Holzenthal 2011, Barcelos-Silva et al. 2012

Polycentropus froehlichi Hamilton \& Holzenthal, 2011

Distribution: Santa Catarina

Notes: Hamilton and Holzenthal 2011 
Polycentropus galharada Hamilton \& Holzenthal, 2011

Distribution: Sao Paulo

Notes: Hamilton and Holzenthal 2011

Polycentropus graciosa Hamilton \& Holzenthal, 2011

Distribution: Parana

Notes: Hamilton and Holzenthal 2011

Polycentropus inusitatus Hamilton \& Holzenthal, 2011

Distribution: Minas Gerais, Rio de Janeiro

Notes: Hamilton and Holzenthal 2011, Dumas and Nessimian 2012

Polycentropus itatiaia Hamilton \& Holzenthal, 2011

Distribution: Minas Gerais, Rio de Janeiro

Notes: Hamilton and Holzenthal 2011

Polycentropus minero Hamilton \& Holzenthal, 2011

Distribution: Minas Gerais

Notes: Hamilton and Holzenthal 2011

Polycentropus paprockii Hamilton \& Holzenthal, 2011

Distribution: Minas Gerais

Notes: Hamilton and Holzenthal 2011

Polycentropus rosalysae Hamilton \& Holzenthal, 2011

Distribution: Minas Gerais, Rio de Janeiro, Sao Paulo

Notes: Hamilton and Holzenthal 2011, Dumas and Nessimian 2012 
Polycentropus santateresae Hamilton \& Holzenthal, 2011

Distribution: Espirito Santo, Minas Gerais

Notes: Hamilton and Holzenthal 2011

Polycentropus soniae Hamilton \& Holzenthal, 2011

Distribution: Parana

Notes: Hamilton and Holzenthal 2011

Polycentropus tripui Hamilton \& Holzenthal, 2011

Distribution: Minas Gerais

Notes: Hamilton and Holzenthal 2011

Polycentropus urubici Holzenthal \& Almeida, 2003

Distribution: Minas Gerais, Parana, Santa Catarina

Notes: Holzenthal and Almeida 2003, Dumas et al. 2010

Polycentropus verruculus Hamilton \& Holzenthal, 2011

Distribution: Minas Gerais, Sao Paulo

Notes: Hamilton and Holzenthal 2011

Polycentropus virginiae Hamilton \& Holzenthal, 2011

Distribution: Minas Gerais

Notes: Hamilton and Holzenthal 2011

Genus Polyplectropus Ulmer, 1905

Notes: Ulmer 1905a, Flint Jr 1968, Bueno-Soria 1990, Chamorro and Holzenthal 2010

Polyplectropus alatespinus Chamorro \& Holzenthal, 2010

Distribution: Minas Gerais, Rio de Janeiro, Sao Paulo

Notes: Chamorro and Holzenthal 2010 
Polyplectropus alleni (Yamamoto), 1967

Distribution: Minas Gerais

Notes: Yamamoto 1967, Blahnik et al. 2004, Chamorro and Holzenthal 2010

Polyplectropus annulicornis Ulmer, 1905

Distribution: Parana, Rio de Janeiro, Rio Grande doSul, Santa Catarina

Notes: Ulmer 1905b, Chamorro and Holzenthal 2010, Dumas and Nessimian 2012

Polyplectropus banksianus Flint, 1971

Distribution: Amazonas

Notes: Flint Jr 1971, Chamorro and Holzenthal 2010

Polyplectropus brachyscolus Flint, 1971

Distribution: Amazonas

Notes: Flint Jr 1971, Chamorro and Holzenthal 2010

Polyplectropus brasilensis Chamorro \& Holzenthal, 2010

Distribution: Rio de Janeiro, Sao Paulo

Notes: Chamorro and Holzenthal 2010

Polyplectropus dubitatus Flint, 1983

Distribution: Minas Gerais, Parana, Santa Catarina

Notes: Flint Jr 1983a, Chamorro and Holzenthal 2010

Polyplectropus elongatus (Yamamoto), 1966

Distribution: Minas Gerais

Notes: Yamamoto 1966, Chamorro and Holzenthal 2010

Polyplectropus flavicornis Ulmer, 1905

Distribution: Minas Gerais, Santa Catarina

Notes: Ulmer 1905a, Chamorro and Holzenthal 2010 
Polyplectropus fuscatus Flint, 1983

Distribution: Santa Catarina

Notes: Flint Jr 1983a, Chamorro and Holzenthal 2010

Polyplectropus gaesum Chamorro \& Holzenthal, 2010

Distribution: Minas Gerais

Notes: Chamorro and Holzenthal 2010

Polyplectropus hollyae Chamorro \& Holzenthal, 2010

Distribution: Minas Gerais

Notes: Chamorro and Holzenthal 2010

Polyplectropus hystricosus Chamorro \& Holzenthal, 2010

Distribution: Minas Gerais, Rio de Janeiro

Notes: Chamorro and Holzenthal 2010, Dumas and Nessimian 2012

Polyplectropus inarmatus Flint, 1971

Distribution: Amazonas

Notes: Flint Jr 1971, Chamorro and Holzenthal 2010

Polyplectropus juliae Chamorro \& Holzenthal, 2010

Distribution: Minas Gerais

Notes: Chamorro and Holzenthal 2010

Polyplectropus matatlanticus Chamorro \& Holzenthal, 2010

Distribution: Minas Gerais, Sao Paulo

Notes: Chamorro and Holzenthal 2010 
Polyplectropus minensium Chamorro \& Holzenthal, 2010

Distribution: Minas Gerais

Notes: Chamorro and Holzenthal 2010

Polyplectropus novafriburgensis Chamorro \& Holzenthal, 2010

Distribution: Rio de Janeiro

Notes: Chamorro and Holzenthal 2010

Polyplectropus petrae Chamorro \& Holzenthal, 2010

Distribution: Minas Gerais

Notes: Chamorro and Holzenthal 2010

Polyplectropus pratherae Chamorro \& Holzenthal, 2010

Distribution: Minas Gerais

Notes: Chamorro and Holzenthal 2010

Polyplectropus rodmani Chamorro \& Holzenthal, 2010

Distribution: Sao Paulo

Notes: Chamorro and Holzenthal 2010

Polyplectropus rondoniensis Chamorro \& Holzenthal, 2010

Distribution: Rondonia

Notes: Chamorro and Holzenthal 2010

Polyplectropus profaupar Holzenthal \& Almeida, 2003

Distribution: Minas Gerais, Parana, Rio de Janeiro, Santa Catarina

Notes: Holzenthal and Almeida 2003, Dumas et al. 2009, Chamorro and Holzenthal 2010, Dumas et al. 2010

Polyplectropus spiculifer Flint, 1971

Distribution: Amazonas 
Notes: Flint Jr 1971, Chamorro and Holzenthal 2010

Polyplectropus tragularius Chamorro \& Holzenthal, 2010

Distribution: Espirito Santo, Minas Gerais, Sao Paulo

Notes: Chamorro and Holzenthal 2010

Polyplectropus ulmeriana Flint, 1983

Distribution: Santa Catarina

Notes: Flint Jr 1983a, Chamorro and Holzenthal 2010

\section{Sericostomatidae}

Genus Grumicha Müller, 1879

Notes: Müller 1879a, Flint Jr et al. 1999a

Grumicha grumicha (Vallot), 1855

Distribution: Santa Catarina, Sao Paulo

Notes: Vallot 1855, Blahnik et al. 2004

Genus Notidobiella Schmid, 1955

Notes: Schmid 1955

Notidobiella amazoniana Holzenthal \& Blahnik, 2010

Distribution: Amazonas

Notes: Holzenthal and Blahnik 2010

Notidobiella brasiliana Holzenthal \& Blahnik, 2010

Distribution: Sao Paulo

Notes: Holzenthal and Blahnik 2010 


\section{Xiphocentronidae}

Genus Machairocentron Schmid, 1982

Notes: Schmid 1982

Machairocentron falciforme Pes \& Hamada, 2013

Distribution: Amazonas

Notes: Pes et al. 2013

Machairocentron sp.

Distribution: Amazonas

Notes: Schmid 1982, Pes et al. 2005

Genus Xiphocentron Brauer, 1870

Notes: Brauer 1870, Schmid 1982

Xiphocentron (Antillotrichia) ilionea Schmid, 1982

Distribution: Rio de Janeiro, Sao Paulo

Notes: Schmid 1982, Dumas et al. 2009

Xiphocentron (Antillotrichia) sclerothrix Pes \& Hamada, 2013

Distribution: Amazonas, Amapa

Notes: Pes et al. 2013

Xiphocentron (Antillotrichia) steffeni (Marlier), 1964

Distribution: Minas Gerais, Rio de Janeiro, Sao Paulo

Notes: Marlier 1964a, Dumas et al. 2010

Xiphocentron (undetermined) saltuum Müller, 1921

Distribution: Brazil

Notes: Müller 1921 


\section{Discussion}

The number of Trichoptera species recorded from Brazil until September, 2014 is 625 . This number represents a $65.34 \%$ increase in species records since 2004 . Ninety percent of the new records were also new species to science. The number of new records since the first Brazilian Checklist is 252, including 223 new species and 29 new country records. The Hydropsychidae rank first in species richness totaling 124 species, followed by Hydroptilidae with 102 species and Polycentropodidae with 97 species. Despite of their ubiquity, caddisflies remain unrecorded in 4 Brazilian states (AL, MA, RN, TO). There is a clear relation between the concentration of universites and researchers and the number of species recorded for a region. Southeastern Brazil, home of the most and the largest universities in Brazil, ranks first with 330 species recorded followed by the Northern region (211 species) and Southern region (165 species). The regions with the greatest lack of knowledge, and which should be considered priorities for Trichoptera inventories, are the Northeast with 74 species and Midwest with 58 species (Fig. 1).

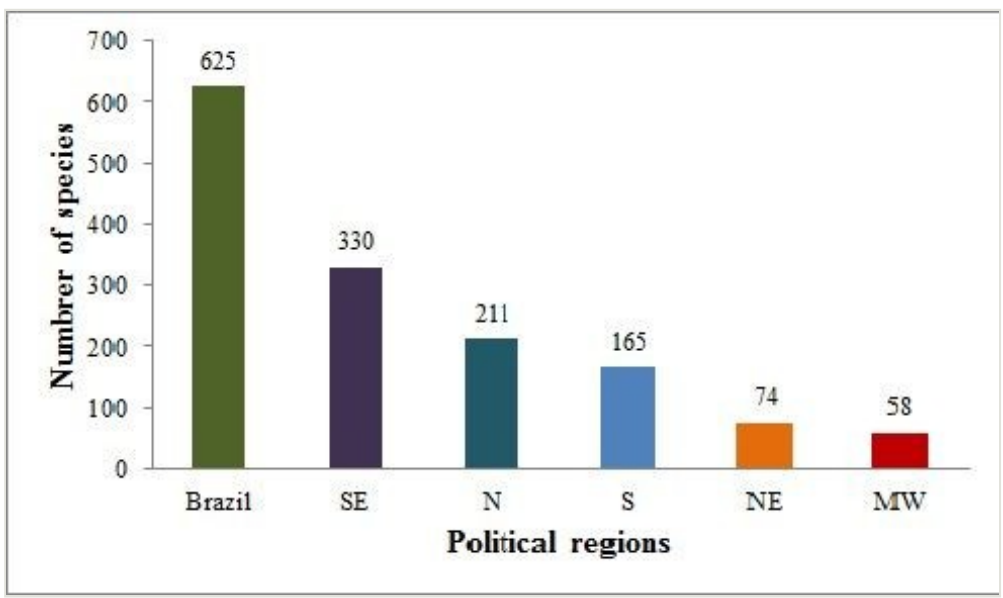

Figure 1.

Number of species by political regions. SE (Southeast), N (North), S (South), NE (Northeast), MW (Midwest). Chart based on data in Suppl. material 1.

The number of regional inventories published since the first Brazilian Trichoptera Checklist is 10 (Blahnik et al. 2004, Ribeiro et al. 2009, Dumas et al. 2009, Nogueira and Cabette 2011, Calor 2011, Dumas and Nessimian 2012, Barcelos-Silva et al. 2012, Souza et al. 2013a, Costa et al. 2014, Quinteiro et al. 2014). It is important to increase the number of regional surveys and publication of regional checklists. The benefits of regional surveys associated with georeferenced data made available online are one of the most important advancements to comprehensive biodiversity data studies (Chavan and Penev 2011). Primary data on Trichoptera biodiversity should be made freely available through inititives like the Global Biodiversity Information System (http://www.gbif.org/). Brazil has undertaken a major initiative of making scientific collections biodiversity information available online 
through the Centro de Referências em Informação Ambiental - CRIA (www.cria.org.br). The numbers acquired by CRIA (August 2014) are impressive and include data from 358 scientific collections, 5,385,443 georreferenced distribution records, and 447,657 species names. The number of Trichoptera records retrieved from CRIA was 6,404. The linkage between scientific journals and such databases should be greatly improved to achieve open access to biodiversity data.

Over six hundred species of Trichoptera have been described for Brazil since the first species was recorded in 1833 (Macrostemum maculatum (Perty) 1833. An average of 2.21 species/year were described between 1833 and the publications of the first comprehensive checklist (Paprocki et al. 2004). Since 2004 the rate of species description has increased dramatically, with an average of 20.2 species/year (Fig. 2).

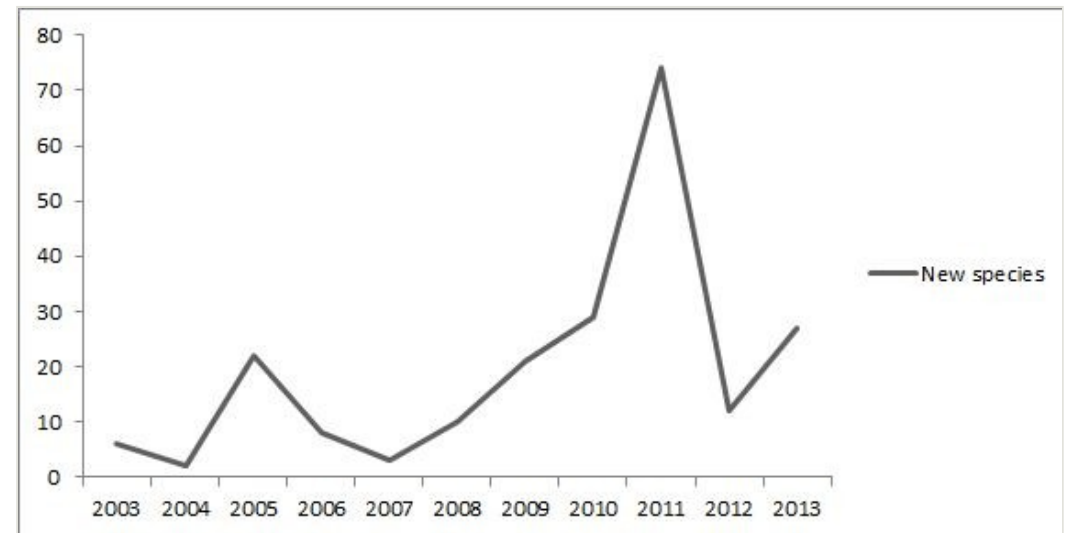

Figure 2.

Number of described species per year since 2003 for Brazil. Chart based on data in Suppl. material 2.

\section{Acknowledgements}

We would like to acknowledge the reviewers of this paper. We also would like to thanks Jordan Biserkov from the Pensoft Writing Tool development team who contributed to the improvement of this article. Part of this work was funded by CEMIG GT 399 project and by TAGMA. 


\section{References}

- $\quad$ Albino JLD, Pes AM, Hamada N (2011) Smicridea (Trichoptera, Hydropsychidae) from 3 Brazilian Amazonian States: New species, larval taxonomy and bionomics. Zootaxa 3113: 1-35.

- $\quad$ Almeida GL, Duarte M (2003) A new species of Dolophilodes (Sortosa) Navás (Trichoptera: Philopotamidae) from Brazil. Proceedings of the Entomological Society Washington 105: 967-969.

- $\quad$ Almeida GL, Flint Jr OS (2002) Five new species of Smicridea McLachlan (Trichoptera: Hydropsychidae) from Brazil. Revista Brasileira de Entomologia 19 (3): 767-775.

- $\quad$ Almeida GL, Marinoni L (2000) Abundância e sazonalidade das espécies de Leptoceridae (Insecta: Trichoptera) capturadas com armadilha luminosa no Estado do Paraná, Brasil. Revista Brasileira de Zoologia 17 (2): 347-359. DOI: 10.1590/ S0101-81752000000200005

- $\quad$ Almeida GL, Marinoni L (2001) Descrição das fêmeas de Chimarra (Curgia) brasiliana (Ulmer) e Chimarra (Curgia) ypsilon Flint (Insecta, Trichoptera, Philopotamidae) do Estado do Paraná, Brasil. Revista Brasileira de Zoologia 18 (3): 971-976. DOI: 10.1590/ S0101-81752001000300029

- $\quad$ Angrisano EB (1994) Contribución al conocimiento de los Trichoptera de Uruguay. I: familias Ecnomidae y Polycentropodidae. Revista de la Sociedad Entomológica Argentina 53: 129-139.

- $\quad$ Angrisano EB (1995) Contribucíon al conocimiento de los Trichoptera del Uruguay. II. Familia Hydroptilidae. Revista Brasileira de Entomologia 39 (3): 501-516.

- $\quad$ Banks N (1904) A list of neuropteroid insects, exclusive of Odonata, from the vicinity of Washington, D.C. Proceedings of the Entomological Society of Washington 6 (4): 201-217.

- Banks N (1905) Descriptions of new Nearctic neuropteroid insects. Transactions of the American Entomological Society 32: 1-20.

- $\quad$ Banks N (1913) Neuropteroid insects from Brazil (The Stanford Expedition to Brazil). Psyche 20: 83-89. DOI: $10.1155 / 1913 / 39865$

- $\quad$ Banks N (1915) New neuropteroid insects, native and exotic. Proceedings of the Academy of Natural Sciences of Philadelphia 66: 608-632.

- $\quad$ Banks N (1920) New neuropteroid insects. Bulletin of the Museum of Comparative Zoology 64 (3): 299-362.

- $\quad$ Banks N (1924) Description of new neuropteroid insects. Bulletin of the Museum of Comparative Zoology 65 (12): 421-455.

- $\quad$ Barbosa FF, Godoy BS, Oliveira LG (2011) Trichoptera Kirby (Insecta) immature fauna from Rio das Almas Basi and Rio Paranã, Goiás State, Brazil, with new records for some genera. Biota Neotropica 11 (4): 21-25.

- $\quad$ Barcelos-Silva P, Pes AMO, Salles FF (2012) Annulipalpia (Insecta: Trichoptera) from the state of Espírito Santo, Brazil. Check List 8 (6): 1274-1279.

- $\quad$ Barcelos-Silva P, Camargos LM, Pes AMO, Salles FF (2013) Six new species of Cernotina Ross, 1938 (Trichoptera: Polycentropodidae) from Brazil. Zootaxa 3669 (2): 115-128. DOI: 10.11646/Zootaxa.3669.2.2 
- Blahnik RJ (1997) Systematics of Chimarrita, a new subgenus of Chimarra (Trichoptera: Philopotamidae). Systematic Entomology 22: 199-243. DOI: 10.1046/

\section{j.1365-3113.1997.d01-39.x}

- Blahnik RJ (1998) A revision of the Neotropical species of the genus Chimarra, subgenus Chimarra (Trichoptera: Philopotamidae). Memoirs of the American Entomological Institute 59: 1-318.

- Blahnik RJ (2002) Systematics of Otarrha, a new Neotropical subgenus of Chimarra (Trichoptera: Philopotamidae). Systematic Entomology 27 (1): 65-130. DOI: 10.1046/ j.0307-6970.2001.00166.x

- Blahnik RJ (2005) Alterosa, a new caddisfly genus from Brazil (Trichoptera: Philopotamidae). Zootaxa 991: 1-60.

- Blahnik RJ, Holzenthal RW (2008) Revision of the Mexican and Central American species of Mortoniella (Trichoptera: Glossosomatidae: Protoptilinae). Zootaxa 1711: 1-72.

- Blahnik RJ, Holzenthal RW (2011) Revision of the austral South American species of Mortoniella (Trichoptera: Glossosomatidae: Protoptilinae). Zootaxa 2851: 1-75.

- Blahnik RJ, Holzenthal RW (2012) New Neotropical species of Chimarra (Trichoptera, Philopotamidae). ZooKeys 184: 1-33. DOI: 10.3897/zookeys.184.2911

- $\quad$ Blahnik RJ, Paprocki H, Holzenthal RW (2004) New Distribution and Species Records of Trichoptera from Southern and Southeastern Brazil. Biota Neotropica 4 (1): 1-6. DOI: 10.1590/S1676-06032004000100009

- $\quad$ Brauer F (1865) Zweiter bericht uber die auf der Weltfahrt der kais. Fregatte Novara gesammelten Neuroptera. Verhandlungen der Kaiserlich-Königlichen ZoologischenBotanischen Gesellschaft in Wien 15: 415-422.

- Brauer F (1870) Ueber Xiphocentron, eine neue Hydropsychidengattung. Verhandlungen der Zoologisch-Botanischen Gesellschaft in Wein 20: 66.

- Bueno-Soria J (1984) Estudios en insectos acuaticos II. revision para México y Centroamerica del genero Hydroptila Dalman, 1819 (Trichoptera: Hydroptilidae). Folia Entomológica Mexicana 59: 79-138.

- Bueno-Soria J (1990) Estudios en insectos acuaticos VIII. revision para Mexico y Centroamerica del genero Polyplectropus Ulmer (Trichoptera: Polyplectopidae). Anales del Instituto de Biología, Universidad Nacional Autónoma de México, Serie Zoología 61 (3): 357-404.

- Bueno-Soria J, Santiago-Fragoso S (1992) Studies in aquatic insects, XI: seven new species of the genus Ochrotirchia (Ochrotrichia) from South America (Trichoptera: Hydroptilidae). Proceedings of the Entomological Society of Washington 94 (4): 439-446.

- $\quad$ Burmeister H (1839) Handbuch der Entomologie, Zweiter Band, Zweite Ubtheilung. Theod. Chr. Friedr. Enslin, Berlin, xii+397-1050 pp.

- $\quad$ Calor AR (2008a) Description of the larvae of Synoestropsis furcata Flint, 1974 (Trichoptera: Hydropsychidae) from central-western region, Brazil. Aquatic Insects 30 (4): 319-325. DOI: 10.1080/01650420802334079

- $\quad$ Calor AR (2008b) A new species of Notalina Mosely, 1936 (Trichoptera: Leptoceridae) from Chapada dos Veadeiros National Park, Goiás state, Brazil. Biotaneotropica 8 (3): 175-178. DOI: $10.1590 /$ S1676-06032008000300016

- Calor AR (2011) Checklist dos Trichoptera (Insecta) do Estado de São Paulo, Brasil. Biota Neotropica 11 (1): 318-328. 
- $\quad$ Calor AR, Holzenthal RW, Amorim DS (2006) Phylogenetic analysis of Notalina (Neonotalina) Holzenthal (Trichoptera: Leptoceridae), with the description of two new species from southeastern Brazil. Zootaxa 1131: 33-48.

- $\quad$ Chamorro ML, Holzenthal RW (2010) Taxonomy and phylogeny of New World Polyplectropus Ulmer, 1905 (Trichoptera: Psychomyioidea: Polycentropodidae) with the description of 39 new species. Zootaxa 2582: 1-252.

- $\quad$ Chavan V, Penev L (2011) The data paper: a mechanism to incentivize data publishing in biodiversity science. BMC Bioinformatics 12 (15): 1-12.

- $\quad$ Costa AM, Quinteiro FB, Calor AR (2014) Capítulo 17: Trichoptera do Semiárido I: Annulipalpia. In: Bravo F, Calor AR (Eds) Artrópodes do Semiárido: biodiversidade e conservação. 1. Printmídia, Feira de Santana, 215-228 pp.

- Dalman JW (1819) Nogra nya insecta-genera beskrifna. Vetenskaps-Akademiens Handlingar 40: 117-127.

- Denning DG, Sykora JL (1968) Three new species of Trichoptera from Brazil. Beiträge zur Neotropischen Fauna 5 (3): 172-177. DOI: 10.1080/01650526809360405

- Dumas LL, Nessimian JL (2008) A new species of Neoatriplectides Holzenthal, 1997 (Insecta: Trichoptera: Atriplectididae), from Brazil, including description of the pupa of the genus. Zootaxa 1773: 63-68.

- Dumas LL, Nessimian JL (2009a) A new species of Leptonema Guérin (Trichoptera: Hydropsychidae) from southeastern Brazil. Zootaxa 2245: 65-68.

- Dumas LL, Nessimian JL (2009b) Description of two new species of Marilia Müller (Trichoptera, Odontoceridae) from southeastern Brazil, including the description of the female of Marilia major Müller. Revista Brasileira de Entomologia 53 (3): 344-348. DOI: 10.1590/S0085-56262009000300006

- Dumas LL, Nessimian JL (2010a) A dwarfish new species of Phylloicus (Trichoptera: Calamoceratidae) from Southeastern Brazil. Zoologia 27 (2): 309-312. DOI: 10.1590/ S1984-46702010000200016

- $\quad$ Dumas LL, Nessimian JL (2010b) A New Long-Horned Caddisfl y in the Genus Triplectides Kolenati (Trichoptera: Leptoceridae) from the Itatiaia Massif, Southeastern Brazil. Neotropical Entomology 39 (6): 949-951. DOI: 10.1590/ S1519-566X2010000600016

- Dumas LL, Nessimian JL (2011) A new species of Cernotina (Trichoptera, Polycentropodidae) from the Atlantic Forest, Rio de Janeiro State, southeastern Brazil. Revista Brasileira de Entomologia 55 (1): 31-34. DOI: 10.1590/ S0085-56262011000100006

- Dumas LL, Nessimian JL (2012) Faunistic Catalog of the Caddisflies (Insecta: Trichoptera) of Parque Nacional do Itatiaia and its Surroundings in Southeastern Brazil. Journal of Insect Science 12 (25): 1-40. DOI: 10.1673/031.012.2501

- Dumas LL, Nessimian JL (2013) New species of the caddisfly genus Alterosa Blahnik 2005 (Trichoptera: Philopotamidae: Philopotaminae) from Brazil. Zootaxa 3609 (1): 26-48. DOI: 10.11646/zootaxa.3609.1.2

- $\quad$ Dumas LL, Calor AR, Nessimian JL (2013) The genus Alterosa Blahnik 2005 (Trichoptera, Philopotamidae, Philopotaminae) in northeastern Brazil, including the description of three new species and an identification key for the genus. ZooKeys 317: 1-15. DOI: 10.3897/zookeys.317.5437 
- Dumas LL, Jardim GA, Santos APM, Nessimian JL (2009) Tricópteros (Insecta: Trichoptera) do Estado do Rio de Janeiro: Lista de espécies e novos registros. Arquivos do Museu Nacional, Rio de Janeiro 67: 355-376.

- Dumas LL, Santos AMP, Jardim GA, Ferreira Jr N, Nessiman JL (2010) Insecta, Trichoptera: New records from Brazil and other distributional notes. CheckList 6 (1): 7.

- Eaton AE (1873) On the Hydroptilidae, a family of the Trichoptera. Transactions of the Entomological Society of London 2: 125-149.

- $\quad$ Edwards SW, Arnold CR (1961) The caddis flies of the San Marcos river. Texas Journal of Science 13 (4): 398-415.

- Emerson BC, Cicconardi F, Fanciulli PP, Shaw PJA (2011) Phylogeny, Phylogeography, Phylobetadiversity and the molecular analysis of biological communities. Philosophical Transactions of the Royal Society 366: 2391-2402. DOI: 10.1098/rstb.2011.0057

- $\quad$ Erichson WF (1848) Insecten. In: Schomburgk R (Ed.) Reisen in Britisch-Guiana in den Jahren 1840-1844. 3. J.J. Weber, Leipzig, 553-617 pp.

- $\quad$ Fischer FCJ (1970) Änderung einiger präokkupierten Namen in der Ordnung Trichoptera. Entomologische Berichten 30: 242-243.

- $\quad$ Flint Jr OS (1964) The caddisflies (Trichoptera) of Puerto Rico. University of Puerto Rica, Agricultural Experiment Station, Technical Paper 40: 1-80.

- $\quad$ Flint Jr OS (1967) Studies of Neotropical Caddiflies II, Trichoptera collected by Prof. J. Illies in the Chilean subregion. Beiträge zur Neotropischen Fauna 5 (1): 45-68. DOI: 10.1080/01650526709360395

- $\quad$ Flint Jr OS (1968) Bredin-Archbold-Smithsonian Biological Survey of Dominica, 9. The Trichoptera (Caddisflies) of the Lesser Antilles. Proceedings of the United States National Museum 125 (3665): 1-86. DOI: 10.5479/si.00963801.125-3665.1

- $\quad$ Flint Jr OS (1969a) Studies of Neotropical caddis flies, IX: new genera and species from the Chilean subregion. Proceedings of the Entomological Society of Washington 71 (4): 497-514.

- $\quad$ Flint Jr OS (1969b) Studies of Neotropical caddis flies, VIII: the immature stages of Barypenthus claudens (Trichoptera: Odontoceridae). Proceedings of the Entomological Society of Washington 71 (1): 24-28.

- $\quad$ Flint Jr OS (1970) Studies of Neotropical cddisflies, X: Leucotrichia and related genera from North and Central America (Trichoptera: Hydroptilidae). Smithsonian Contributions to Zoology 60: 1-64. DOI: 10.5479/si.00810282.60

- $\quad$ Flint Jr OS (1971) Studies of Neotropical caddisflies, XII: Rhyacophilidae, Glossosomatidae, Philopotamidae, and Psychomyiidae from the Amazon Basin (Trichoptera). Amazoniana 3 (1): 1-67.

- $\quad$ Flint Jr OS (1972) Studies of Neotropical caddisflies, XIV: on a collection from northern Argentina. Proceedings of the Biological Society of Washington 85 (17): 223-248.

- $\quad$ Flint Jr OS (1973) Studies of Neotropical caddisflies, XVI: the genus Austrotinodes (Trichoptera: Psychomyiidae). Proceedings of the Biological Society of Washington 86 (11): 127-142.

- $\quad$ Flint Jr OS (1974a) Studies of Neotropical Caddsiflies, XV: The Trichoptera of Surinam. Studies on the Fauna of Suriname and other Guyanas 14 (55): 1-151.

- $\quad$ Flint Jr OS (1974b) Studies of Neotropical caddisflies, XVIII: new species of Rhyacophilidae and Glossosomatidae (Trichoptera). Smithsonian Contributions to Zoology 169: 1-30. 
- $\quad$ Flint Jr OS (1974c) Studies of Neotropical caddisflies, XVII: the genus Smicridea from North and Central America (Trichoptera: Hydropsychidae). Smithsonian Contributions to Zoology 167: 1-65.

- $\quad$ Flint Jr OS (1978) Studies of Neotropical Caddisflies XXII: Hydropsychidae of the Amazon Basin (Trichoptera). Amazoniana 6 (3): 373-421.

- Flint Jr OS (1980) Studies on Neotropical caddisflies, XXVI: new species from Argentina (Trichoptera). Revista de la Sociedad Entomológica Argentina 39: 137-142.

- Flint Jr OS (1981) Studies of Neotropical Caddisflies, XXVIII: the Trichoptera of the Río Limón Basin, Venezuela. Smithsonian Contributions to Zoology 330: 1-61. DOI:

\subsection{9/si.00810282.330}

- $\quad$ Flint Jr OS (1982a) Studies of Neotropical caddisflies, XXXI: five new species from Argentina (Trichoptera). Entomological News 93 (2): 43-47.

- $\quad$ Flint Jr OS (1982b) Trichoptera of the area Platense. Biologia Acuatica 2 (213): 1-70.

- $\quad$ Flint Jr OS (1983a) Studies of Neotropical caddisflies, XXXIV: the genus Plectromacronema (Trichoptera: Hydropsychidae). Proceedings of the Biological Society of Washington 96 (2): 225-237. DOI: 10.5479/si.00810282.377

- $\quad$ Flint Jr OS (1983b) Studies of Neotropical caddisflies, XXXIII: new species from austral South America (Trichoptera). Smithsonian Contributions to Zoology 377: 1-11. DOI: 10.5479/si.00810282.377

- $\quad$ Flint Jr OS (1989) Studies of Neotropical Caddisflies, XXXIX: The Genus Smicridea in the Chilean Subregion (Trichoptera: Hydropsychidae). Smithsonian Contributions to Zoology 472: 1-45. DOI: 10.5479/si.00810282.472

- Flint Jr OS (1991) Studies of Neotropical caddisflies, XLIV: on a collection from Ilha de Maraca, Brazil. Acta Amazonica 21 (1): 63-83.

- Flint Jr OS (1992) Studies of Neotropical caddisflies, XXXVIII: a review of the classification and biology of the Neotropical microcaddisflies, with the description of a new genus (Trichoptera: Hydroptilidae: Leucotrichiini). In: Quintero D, Aiello A (Eds) Insects of Panama and Mesoamerica: Selected Studies. Oxford University Press, Oxford, 525-531 pp.

- $\quad$ Flint Jr OS (1996) The Trichoptera Collected on the Expeditions to Parque Manu, Madre de Dios, Peru. In: Wilson DE, Sandoval A (Eds) MANU: The Biodiversity of Southeastern Peru. Smithsonian Institution Press, Washington, DC, 369-430 pp.

- Flint Jr OS (1998) Studies fo Neotropical Caddsiflies, LIII: A Taxonomic Revision of the Subgenus Curgia of the genus Chimarra (Trichoptera: Philopotamidae). Smithsonian Contributions to Zoology 594: 1-131. DOI: $10.5479 /$ si.00810282.594

- Flint Jr OS (2008) Studies of Neotropical Caddisflies, LXI: New Species of Leptonema GuéRin (Trichoptera: Hydropsychidae). Proceedings of the Entomological Society Washington 110 (2): 456-469. DOI: 10.4289/07-042.1

- $\quad$ Flint Jr OS, Bueno-Soria J (1982) Studies of neotropical caddisflies, XXXII: the immature stages of Macronema variipenne Flint \& Bueno. with the division of Macronema by the resurrection of Macrostemum (Trichoptera: Hydropsychidae). Proceedings of the Biological Society of Washington 95 (2): 358-370.

- $\quad$ Flint Jr OS, Denning DG (1989) Studies of Neotropical caddisflies, XLI: new species and records of Austrotinodes (Trichoptera: Psychomyiidae). Pan-Pacific Entomologist 65 (2): 108-122. DOI: 10.5479/si.00810282.472

- $\quad$ Flint Jr OS, Harris SC (1992) Studies of Neotripical Caddisflies, XLII: Taraxitrichia amazonensis, a new genus and species of microcaddisfly from Venezuela Trichoptera: 
Hydroptilidae). Proceedings of the 6th International Symposium on Trichoptera 1: 411-414.

- $\quad$ Flint Jr OS, Holzenthal RW, Harris SC (1999a) Catalog of the Neotropical Caddisflies (Insecta: Trichoptera). Special Publication, Ohio Biological Survey, Columbus, Ohio, $239 \mathrm{pp}$.

- $\quad$ Flint Jr OS, Holzenthal RW, Harris SC (1999b) Nomenclatural and systematic changes in the Neotropical caddisflies. Insecta Mundi 13 (1): 73-84.

- $\quad$ Flint OS, McAlpine JF, Ross HH (1987) A revision of the genus Leptonema Guerin (Trichoptera: Hydropsychidae: Macronematinae). Smithsonian Contributions to Zoology 450: 1-193. DOI: $10.5479 /$ si.00810282.450

- Fonseca G, Benson PJ (2003) Biodiversity Conservation Demands Open Access. PLoS Biol 1 (2): 163-165. DOI: 10.1371/journal.pbio.0000046

- $\quad$ França D, Paprocki H, Calor AR (2013) The genus Macrostemum Kolenati 1859 (Trichoptera: Hydropsychidae) in the Neotropical Region: Description of two new species, taxonomic notes, distributional records and key to males. Zootaxa 3716 (3): 301-335.

- $\quad$ Guahyba RR (1991) Estágios imaturos de Anchitrichia duplifurcata Flint, 1983 (Trichoptera, Hydroptilidae). Revista Brasileira de Entomologia 35 (1): 121-125.

- Guérin-Méneville FE (1843) Insectes. Iconographie du Régne Animal du Cuvier. J.B. Baillière, Paris.

- Hagen HA (1861) Synopsis of the Neuroptera of North America with a list of the South American species. Smithsonian Miscellaneous Collections 347: 248-299.

- Hamilton SW, Holzenthal RW (2011) Twenty-four new species of Polycentropus (Trichoptera, Polycentropodidae) from Brazil. ZooKeys 76: 1-53.

- Harris S, Holzenthal RW (1992) Hydroptilidae (Trichoptera) of Costa Rica: the genus Oxyethira Eaton. Journal of the New York Entomological Society 100: 155-177.

- Harris SC (1990) New species of Neotrichia (Trichoptera: Hydroptilidae) from Central and South America. Journal of the New York Entomological Society 98 (2): 246-260.

- $\quad$ Harris SC, Armitage BJ (1997) New member of the Chilean genus Nothotrichia from North America (Trichoptera: Hydroptilidae). In: Holzenthal RW, Flint Jr OS (Eds) Proceedings of the 8th International Symposium on Trichoptera. Ohio Biological Survey, Columbus, Ohio, 123-128 pp.

- Harris SC, Davenport LJ (1999) New species of Hydroptilidae (Trichoptera) from the Amazon Region of Northeastern Peru. Proceedings of the Entomological Society Washington 101 (1): 26-38.

- Harris SC, Flint Jr OS (2002) New Alisotrichia (Trichoptera: Hydroptilidae) from Central and South America and the Greater Antilles. Proceedings of the Entomological Society of Washington 104 (1): 195-210.

- Harris SC, Holzenthal RW (1994) Hydroptilidae (Trichoptera) of Costa Rica and the Neotropics: Systematics of the genus Byrsopteryx Flint (Stactobiini). Journal of the New York Entomological Society 102 (2): 154-192.

- Harris SC, Holzenthal RW (1999) Hydroptilidae (Trichoptera) of Costa Rica: the genus Hydroptila Dalman. Studies on Neotropical Fauna and Environment 34: 16-51.

- Harris SC, Flint Jr OS, Holzenthal RW (2002a) Review of the Neotropical genus Flintiella (Trichoptera: Hydroptilidae: Stactobiini). Journal of the New York Entomological Society 110 (1): 65-90. 
- Harris SC, Holzenthal RW, Flint Jr OS (2002b) Review of the Neotropical genus Bredinia (Trichoptera: Hydroptilidae: Stactobiini). Annals of Carnegie Museum 71 (1): 13-45.

- Henriques-Oliveira AL, Dumas LL, Nessimian JL (2014) Three new species and new distributional records of Oecetis McLachlan 1877 (Trichoptera: Leptoceridae:

Leptocerinae) from Brazil. Zootaxa 3753 (3): 273-282.

- Henriques-Oliveira AL, Spies MR, Dumas LL (2012) A new species of Notalina Mosely, 1936 (Trichoptera: Leptoceridae) from Southeastern Brazil. Biota Neotropica 12 (4): 130-134.

- Holzenthal RW (1984) Studies in Neotropical Leptoceridae (Trichoptera) I: Achoropsyche, a new genus. In: Morse JC (Ed.) Proceedings of the 4th International Symposium on Trichoptera. Dr. W. Junk, The Hague, 181-184 pp.

- Holzenthal RW (1986) The Neotropical species of Notalina, a southern group of longhorned caddisflies (Trichoptera: Leptoceridae). Systematic Entomology 11: 61-73.

- Holzenthal RW (1988a) Systematics of Neotropical Triplectides (Trichoptera: Leptoceridae). Annals of the Entomological Society of America 81 (2): 187-208.

- Holzenthal RW (1988b) Studies in Neotropical Leptoceridae (Trichoptera), VIII: the genera Atanatolica Mosely and Grumichella Müller (Triplectidinae: Grumichellini). Transactions of the American Entomological Society 114: 71-128.

- Holzenthal RW (1989) Studies in Neotropical Leptoceridae (Trichoptera), IX: a new genus and species from southeastern Brazil. Aquatic Insects 11 (1): 29-32.

- Holzenthal RW (1995) The caddisfly genus Nectopsyche: new gemma group species from Costa Rica and the Neotropics (Trichoptera: Leptoceridae). Journal of the North American Benthological Society 14: 61-83.

- Holzenthal RW (1997) The caddisfly (Trichoptera) family Atriplectididae in the Neotropics. In: Holzenthal RW, Flint Jr. OS (Eds) Proceedings of the 8th International Symposium on Trichoptera. Ohio Biological Survey, Columbus, Ohio, 157-165 pp.

- Holzenthal RW, Almeida GL (2003) New species of Polycentropodidae (Trichoptera) from southeastern and southern Brazil. Proceedings of the Entomological Society of Washington 105 (1): 22-29.

- Holzenthal RW, Blahnik RJ (2010) Systematics of the Neotropical caddisfly genus Notidobiella Schmid (Trichoptera, Sericostomatidae), with the description of 3 new species. ZooKeys 71: 23-47.

- Holzenthal RW, Flint Jr OS (1995) Studies of Neotropical caddisflies, LI: systematics of the Neotropical caddisfly genus Contulma (Trichoptera: Anomalopsychidae).

Smithsonian Contributions to Zoology 575: 1-59.

- Holzenthal RW, Harris SC (2002) New species of Nothotrichia Flint (Trichoptera: Hydroptilidae) from Brazil and Costa Rica. Proceedings of the Entomological Society of Washington 104 (1): 106-110.

- Holzenthal RW, Pes AM (2004) A new genus of long-horned caddisfly from the Amazon basin (Trichoptera: Leptoceridae: Grumichellini). Zootaxa 621: 1-16.

- Holzenthal RW, Robertson DR (2006) Four new species of Contulma from South America (Trichoptera: Anomalopsychidae). Zootaxa 1335: 49-59.

- Huamantico AA, Dumas LL, Nessimian JL (2005) Description of larva and pupa of Phylloicus abdominalis Ulmer, 1905 (Trichoptera: Calamoceratidae). Zootaxa 1039: 19-26. 
- Huamantinco AA, Nessimian JL (2004a) Description of the larva and pupa of Antarctoecia brasiliensis Huamantinco \& Nessimian, 2003 (Trichoptera, Limnephilidae). Zootaxa 490 (1): 1-8.

- Huamantinco AA, Nessimian JL (2004b) A New Neotropical Genus and Species of Odontocerinae (Trichoptera: Odontoceridae) from Southeastern Brazil. Aquatic Insects 26: 281-288.

- Huamantinco AA, Nessimian J,L (2003) A New Species of Antarctoecia Ulmer, 1907 (Trichoptera: Limnephilidae) from Southeastern Brazil. Aquatic Insects 25 (3): 225-231. Jardim GA, Dumas LL (2012) A new species of the genus Alterosa (Trichoptera: Philopotaminae) from Southeastern Brazil. Revista de Biología Tropical 60 (2): 577-580.

- Jardim GA, Nessimian JL (2011) A new species of Contulma Flint (Trichoptera, Anomalopsychidae) from southeastern Brazil. Revista Brasileira de Entomologia 55 (2): 226-228. DOI: 10.1590/S0085-56262011005000014

- Jardim GA, Dumas LL, Nessiman JL (2010) Three new species of Leptonema Guérin (Trichoptera: Hydropsychidae) from southeastern Brazil. Zootaxa 2654: 52-60.

- Johanson KA (1999) Description of two new Neotropical Helicopsyche (Trichoptera: Helicopsychidae). Aquatic Insects 21 (2): 127-132. DOI: 10.1076/aqin.21.2.127.4535 Johanson KA (2003) Revision of the Neotropical caddisfly subgenus Helicopsyche (Cochliopsyche) (Trichoptera, Helicopsychidae), with descriptions of twelve new species. Insect Systematics \& Evolution 34 (4): 381-414. DOI:

10.1163/187631203X00036

- Johanson KA, Malm T (2006) Seven new Helicopsyche (Feropsyche) Johanson, 2002 from the Neotropical region and Nearctic Mexico (Insecta: Trichoptera:

Helicopsychidae). Zootaxa 1208: 1-24.

- $\quad$ Kelley RW (1983) New Neotropical species of Oxyethira (Trichoptera: Hydroptilidae). Proceedings of the Entomological Society Washington 85: 41-54.

- Kloet GS, Hincks WD (1944) Nomenclatorial notes on two generic names in the Trichoptera. Entomologist 77: 97.

- Kolenati FA (1859) Genera et Species Trichopterorum, Pars, Altera. Nouveaux Mémoires de la Société Impérialedes Naturalistes de Moscou 11: 141-296.

- Machado ABM (1957) Helicopsyche planorboides n. sp. (Trichoptera Helicopsychidae) and its mimetic relationship to planorbids (Mollusca Pulmonata). Anais da Academia Brasileira de Ciencias 29 (1): 193-200.

- Marinoni L, Almeida GL (2000) Abundância e sazonalidade das espécies de Hydropsychidae (Insecta, Trichoptera) capturadas em armadilha luminosa no Estado do Paraná, Brasil. Revista Brasileira de Zoologia 17 (1): 283-299. DOI: 10.1590/ S0101-81752000000100025

- Marlier G (1964a) Trichoptères de l'Amazonie recueillis par le Professeur H. Sioli. Memoires I'Institut Royal des Sciences Naturelles de Belgique 2 (76): 1-167.

- Marlier G (1964b) Sur trois trichopteres nouveaux recueillis en Amerique du Sud par le Professeur J. Illies. Bulletin de I'Institut Royal des Sciences Naturalles de Belgique 40 (6): 1-15.

- Martins-Silva MJ, Engel DW, Rocha FM, Araújo J (2008) Imaturos de Trichoptera na Bacia do Rio Paranã, GO, com Novos Registros de Gênero. Netropical Entomology 37 (6): 735-738.

- Martynov AV (1912) On two collections of Trichoptera from Peru. Annuaire du Musée Zoologique de l'Académie Impériale des Sciences de Saint Pétersbourg 17: 1-40. 
- McLachlan R (1871) On new forms, etc., of extra-European trichopterous insects. Journal of the Linnean Society of London, Zoology 11: 98-141. DOI: 10.1111/ j.1096-3642.1870.tb02011.x

- McLachlan R (1877) A monographic revision and synopsis of the Trichoptera of the European fauna. Part 6. John van Voorst, London, 281-348, plates 32-37 pp.

- Morton KJ (1905) North American Hydroptilidae. Bulletin of the New York State Museum 86: 63-75.

- Mosely ME (1933) A revision of the genus Leptonema (Trichoptera). British Museum (Natural History), London 1: 1-69.

- $\quad$ Mosely ME (1934a) Some new exotic Trichoptera. Stylops 3 (6): 139-142.

- Mosely ME (1934b) New exotic Hydroptilidae. Transactions of the Royal Entomological Society of London 82 (1): 137-163. DOI: 10.1111/j.1365-2311.1934.tb00031.x

- Mosely ME (1936) A revision of the Triplectidinae, a subfamily of the Leptoceridae (Trichoptera). Transactions of the Royal Entomological Society of London 85 (3): 91-130. DOI: 10.1111/j.1365-2311.1936.tb00241.x

- Mosely ME (1937) Mexican Hydroptilidae (Trichoptera). Transactions of the Royal Entomological Society of London 86 (10): 151-189. DOI: 10.1111/ j.1365-2311.1937.tb00242.x

- Mosely ME (1939) The Brazilian Hydroptilidae (Trichoptera). Novitates Zoologicae 41: 217-239.

- Müller F (1879a) Notes on the cases of some South Brazilian Trichoptera. Transactions of the Royal Entomological Society of London 4 (2): 131-144.

- $\quad$ Müller F (1879b) Über Phryganiden (letters to his brother). Zoologischer Anzeiger 2: 38-407.

- Müller F (1880) Sobre as casas construidas pelas larvas de insectos Trichopteros da Provincia de Santa Catharina. Archivos do Museu Nacional, Rio de Janeiro 3 (1878): 99-214.

- Müller F (1888) Larven und Mucken und Haarflügler mit zweierlei abwechselnd tätigen atemwerkzeugen. Entomologische Nachrichten 14 (18): 273-277.

- Müller F (1921) Briefe un noch nicht veröffentliche Abhandlungen aus dem nachlass 1854-1897. In: Möller A (Ed.) Fritz Müller: Werke, Briefe und Leben, 2. G. Fischer, Jena, 383-642 pp.

- $\quad$ Mülller F (1885) Wie Entsteht die Gleiderung der Insektenfuhler? Kosmos 17: 201-204.

- Navás L (1916a) Neurópteros Sudamericanos. Brotéria, Série Zoológica 14: 14-35.

- Navás L (1916b) Neuroptera Nova Americana. Series I, II. Memorie della Pontificia Accademia Romana dei Nuovi Lincei 2 (2): 59-80.

- $\quad$ Navás L (1917) Neurópteros nuevas o poco conocidos (novena serie). Memorias de la Real Academia de Ciencias y Artes de Barcelona, Tercera Epoca 13: 393-406.

- Navás L (1918) Algunos insectos de la República Argentina, Serie I. Revista de la Real Academia de Ciencias Exactas, Físicas y Naturales de Madrid, 2a Serie 16: 491-504.

- Navás L (1920) Insectos Sudamericanos (1a, 2a y 3a serie). Anales de la Sociedad Cientifica Argentina 90: 33-72.

- Navás L (1924) Quelques insectos de l'Amérique Méridionale. Annales de la Société Scientifique de Bruxelles, Documents et Comptes Rendus 43: 249-254.

- Navás L (1930) Insectos Neotrópicos, 6 serie (1). Revista Chilena de Historia Natural 34: 62 34: 62-75.

- $\quad$ Navás L (1931) Insectos del Brasil 4a serie. Revista do Museu Paulista 17 (1): 455-458. 
- Navás L (1932a) Décadas de Insecta Nuevos. Década 20. Brotéria, Ciencias Naturais 1: $74-85$.

- Navás L (1932b) Insectos suramericanos, Quinta Serie. Revista de la Academia de Ciencias de Madrid 29: 53-66.

- Neboiss A (1993) Revised definitions of the genera of Nyctiophylax Brauer and Paranyctiophylax Tsuda (Trichoptera: Polycentropodidae). In: Otto C (Ed.) Proceedings of the 7th International Symposium on Trichoptera. Backhuys Publishers, Leiden, The Netherlands, 107-111 pp.

- $\quad$ Nogueira DS, Cabette HSR (2011) Novos registros e notas sobre distribuição geográfica de Trichoptera Kirby, 1813 (Insecta) do Estado do Mato Grosso, Brasil. Biota Neotropica 11 (2): 347-355. DOI: 10.1590/S1676-06032011000200033

- $\quad$ Oláh J, Johanson KA (2011) New Neotropical Hydroptilidae (Trichoptera). Annales Historico-Naturales Musei Nationalis Hungarici 103: 117-255.

- Paprocki H, Holzenthal RW (2002) A review of the Brazilian genus Barypenthus Burmeister (Trichoptera: Odontoceridae). Nova Supplementa Entomologica (Proceedings of the 10th International Symposium on Trichoptera) 15 (2002): 223-230.

- Paprocki H, Holzenthal RW, Blahnik RJ (2004) Checklist of the Trichoptera (Insecta) of Brazil I. Biota Neotropica 4 (1): 1-22. DOI: 10.1590/S1676-06032004000100008

- $\quad$ Perty JAM (1833) Insecta Brasiliensia. In: Martius KFP (Ed.) Delectus Animalium Articulatorum. Impensis Editoris, Monachii, 1-224 pp.

- $\quad$ Pes AMO, Hamada N (2003) The occurrence of Taraxitrichia Flint \& Harris, 1992 (Trichoptera: Hydroptilidae) in Brazil, with description of the final larval stage. Zootaxa 328: 1-7.

- Pes AMO, Hamada N (2004) Ceratotrichia Flint, 1992 (Trichoptera: Hydroptilidae) larval and pupal description and new genus records for Brazil. Entomotropica 19 (1): 31-37.

- $\quad$ Pes AMO, Hamada N, Nessimian JL (2005) Chaves de identificação de larvas para famílias e gêneros de Trichoptera (Insecta) da Amazônia Central, Brasil. 49 (2): 181-204.

- $\quad$ Pes AMO, Hamada N, Nessimian JL, Soares CC (2013) Two new species of Xiphocentronidae (Trichoptera) and their bionomics in Central Amazonia, Brazil. Zootaxa 3636 (4): 561-574. DOI: 10.11646/zootaxa.3636.4.4

- $\quad$ Pictet FJ (1836) Description de quelques nouvelles espèces de Névroptères, du Musée de Genève. Mémoires de La Société de Physique et d'Histoire Naturelle de Gèneve 7: 399-404.

- $\quad$ Prather AL (2003) Revision of the Neotropical caddisfly genus Phylloicus (Trichoptera: Calamoceratidae). Zootaxa 275: 1-214.

- $\quad$ Quinteiro FB, Calor AR (2012) A new species of Oecetis McLachlan, 1877 (Trichoptera: Leptoceridae) from Southeast Brazil: Validation of an unplished species. Zootaxa 3442: 53-61.

- $\quad$ Quinteiro FB, Calor AR, Froehlich CG (2011) A new species of Phylloicus Müller, 1880 (Trichoptera: Calamoceratidae), from southeastern Brazil, including descriptions of larval and pupal stages. Zootaxa 2748: 38-46.

- $\quad$ Quinteiro FB, Costa AM, Calor AC (2014) Capítulo 18: Trichoptera do Semiárido II: Integripalpia. In: Bravo F, Calor AR (Eds) Artrópodes do Semiárido: biodiversidade e conservação. 1. Printmídia, Feira de Santana, 229-244 pp.

- Rambur MP (1842) Histoire naturelle des insectes névroptères. Librairie Encyclopédique de Roret, Paris, xviii $+534,12$ plates pp. 
- $\quad$ Ribeiro JMF, Magalhães C, Rafael JA, Henrique AL (2009) Catalogue of type specimens of the Collection of Invertebrates of Instituto Nacional de Pesquisas da Amazônia, Manaus, Brazil. III. Hexapoda: Isoptera, Mantodea, Mecoptera, Orthoptera, Plecoptera, Trichoptera and Zoraptera. Revista Brasileira de Entomologia 53 (1): 32-35.

- Robertson DR, Holzenthal RW (2005) The Neotropical caddisfly genus Tolhuaca (Trichoptera: Glossosomatidae). Zootaxa 1063: 53-68.

- Robertson DR, Holzenthal RW (2006) The Neotropical caddisfly genus Canoptila (Trichoptera: Glossosomatidae). Zootaxa 1272: 45-59.

- $\quad$ Robertson DR, Holzenthal RW (2011) Revision of the Neotropical caddisfly genus Itauara Müller, 1888 (Trichoptera, Glossosomatidae). ZooKeys 114: 41-100. DOI: 10.3897/zookeys.114.1405

- $\quad$ Ross HH (1938) Descriptions of Nearctic caddisflies (Trichoptera) with special reference to the Illinois species. Bulletin of the Illinois Natural History Survey 21 (4): 101-183.

- $\quad$ Ross HH (1947) Description and records of North American Trichoptera, with synoptic notes. Trans Amer Entomol Soc 73: 125-168.

- Ross HH (1953) Additional material on the phylogeny and dispersal of Atopsyche (Trichoptera, Rhyacophilidae). Journal of the Washington Academy of Sciences 43 (9): 287-293.

- $\quad$ Ross HH (1956a) Evolution and Classification of the Mountain Caddisflies. University of Illinois Press, Urbana, $213 \mathrm{pp}$.

- $\quad$ Ross HH (1956b) New species of Helicopsyche from the western hemisphere (Trichoptera, Helicopsychidae). Journal of the Washington Academy of Sciences 46 (12): 397-401.

- $\quad$ Ross HH, King EW (1952) A key to the World genera of the caddisfly tribe 'Hydrobiosini' (Trichoptera, Rhyacophilidae). Acta Zool Lilloana 12: 177-204.

- $\quad$ Santos APM (2011) Four new species of the microcaddisfly genus Alisotrichia Flint (Trichoptera: Hydroptilidae) from southeastern Brazil. Zootaxa 3112: 59-68.

- $\quad$ Santos APM, Holzenthal RW (2012) Three new species of Atopsyche Banks (Trichoptera, Hydrobiosidae) from Brazil. ZooKeys 207: 65-78. DOI: $10.3897 /$ zookeys.207.3419

- $\quad$ Santos APM, Nessimian JL (2008) Five new species of Cernotina Ross (Trichoptera: Polycentropodidae) from Central Amazonia, Brazil. Zootaxa 1899: 25-33.

- $\quad$ Santos APM, Nessimian JL (2009a) A new species of microcaddisfly genus Flintiella Angrisano (Trichoptera: Hydroptilidae) from Amazonas State, Brazil. Zootaxa 2004: 65-68. DOI: 10.1590/S0044-59672009000300030

- Santos APM, Nessimian JL (2009b) New species and records of Neotrichia (Trichoptera: Hydroptilidae) from Central Amazonia, Brazil. Zoologia 26 (4): 758-768. DOI: $10.1590 /$ S1984-46702009000400022

- Santos APM, Nessimian JL (2009c) A new species of Protoptila Banks (Trichoptera: Glossosomatidae: Protoptilinae) from Brazil. Acta Amazonica 39 (3): 723-726. DOI: 10.1590/S0044-59672009000300030

- Santos APM, Nessimian JL (2009d) New species and records of Chimarra Stephens (Trichoptera, Philopotamidae) from Central Amazonia, Brazil. Revista Brasileira de Entomologia 53 (1): 23. DOI: 10.1590/S0085-56262009000100006

- Santos APM, Nessimian JL (2010a) Description of a new species of Byrsopteryx (Trichoptera: Hydroptilidae) from Rio de Janeiro State, Brazil, including its immature stages. Zootaxa 2668: 44-54. 
- Santos APM, Nessimian JL (2010b) A remarkable new species of Phylloicus (Trichoptera: Calamoceratidae) from Central Amazonia, Brazil. Aquatic Insects 32 (4): 321-326. DOI: $10.1080 / 01650424.2010 .508046$

- $\quad$ Santos APM, Nessimian JL (2010c) The occurrence of the microcaddisfly Costatrichia (Trichoptera: Hydroptilidae: Hydroptilinae) in Brazil with description of two new species. Zoologia 27 (5): 837-843. DOI: 10.1590/S1984-46702010000500022

- Santos APM, Henriques-Oliveira AL, Nessimian JL (2009) New species and records of Oxyethira Eaton (Trichoptera: Hydroptilidae) from Amazonas State, Brazil. Zootaxa 2169: 35-44.

- Santos APM, Jardim GA, Nessimian JL (2011) Three new species of microcaddisflies (Trichoptera: Hydroptilidae) from Brazil. Zoologia 28 (6): 812-818. DOI: 10.1590/ S1984-46702011000600015

- $\quad$ Santos APM, Takiya DM, Nessimian JL (2013) Two new species of Costatrichia (Trichoptera: Hydroptilidae: Leucotrichiinae). Zoologia 20 (4): 447-450. DOI: 10.1590/ S1984-46702013000400012

- $\quad$ Sattler W (1963) Eine neue Triplectides-Art (Leptoceridae, Trichoptera) aus dem brasilianischen Amazonasgebiet, ihre Metamorphosestadien und Bemerkungen zu ihrer Biologie. Beiträge zur Neotropischen Fauna 3: 20-33. DOI:

\subsection{0/01650526309360364}

- $\quad$ Sattler W, Sykora JL (1977) Über eine, besonders durch ihr Bauinstinkt, merkwürdige neotropische Köcherfliege - Leucotrichia brasiliana n. sp. Amazonia 6 (2): 137-255.

- Schmid $F(1955)$ Contribution à la connaissance des Trichoptères néotropicaux. Mémoires de la Société Vaudoise des Sciences Naturelles 11 (69): 117-160.

- Schmid F (1964) Contribution à l'étude des Trichoptères néotropicaux V. Tijdschrift voor Entomologie 107 (6): 307-339.

- $\quad$ Schmid F (1982) La famille des Xiphocentronides (Trichoptera: Annulipalpia). Memoires de la Société Entomologique du Canada 121: 1-126.

- $\quad$ Schmid F (1989) Les Hydrobiosides (Trichoptera, Annulipalpia). Bulletin de I'Institute Royal des Sciences Naturelles de Belgique, Entomologie 59: 1-154.

- Siebold CTE (1856) Wahre Parthenogenesis bei Schmetterlingen und Bienen. Wilhelm Engelmann, Leipzig, vi+144 pp.

- $\quad$ Silva RAL, Santos APM, Nessimian JL (2014) Helicopsyche (Feropsyche) timbira sp. nov. (Trichoptera: Helicopsychidae), a new species from southeastern Brazil. Zootaxa 3847 (3): 446-448. DOI: 10.11646/zootaxa.3847.3.9

- $\quad$ Souza WRM, Santos APM, Takiya DM (2014) First records of Ochrotrichia Mosely, 1934 (Trichoptera: Hydroptilidae) in Northeastern Brazil: Five new species and two new geographical records. Zootaxa 3852 (2): 273. DOI: 10.11646/zootaxa.3852.2.6

- Souza WRM, Lima LRC, Pes AMO, Pinheiro U (2013a) Trichoptera (Insecta) from Pernambuco State, northeastern Brazil. Journal of Natural History 1: 1-10.

- Souza WRM, Santos APM, Lima LRC, Pinheiro U (2013b) A new species and new records of microcaddisflies (Trichoptera: Hydroptilidae) from northeastern Brazil. Zootaxa 3700 (4): 583-587. DOI: 10.11646/zootaxa.3700.4.6

- Spies MR, Froehlich C (2009) Inventory of caddisflies (Trichoptera: Insecta) of the Campos do Jordão State Park, São Paulo State, Brazil. Biota Neotropica 9 (4): 211-218. DOI: 10.1590/S1676-06032009000400021

- Spies MR, Froehlich CG, Kotzian CB (2006) Composition and diversity of Trichoptera (Insecta) larvae communities in the middle section of the Jacuí river and some 
tributaries, State of Rio Grande do Sul, Brazil. Iheringia Série Zoologia 96 (4): 389-398. DOI: $10.1590 / S 0073-47212006000400001$

- $\quad$ Stephens JF (1829) A Systematic Catalogue of British Insects: Being an Attempt to Arrange all the Hitherto Discovered Indigenous Insects in Accordance with their Natural Affinities. Containing also the References to every English Writer on Entomology, and to the Principal Foreign Authors. With all the Published British Genera to the Present Time. Part 1. Insecta Mandibulata. [Trichoptera pages 316-323]. Baldwin and Cradock, London, xxxiv+416 pp.

- $\quad$ Swainson W (1840) A Treatise on Malacology: or the Natural Classification of Shells and Shell-fish. Longman, London, viii+419 pp.

- $\quad$ Sykora JL (1998) New species of Cernotina Ross (Insecta: Trichoptera: Polycentropodidae) from the Amazon Basin in northeastern Peru and northern Brazil. Annals of Carnegie Museum 67 (2): 95-104.

- Thienemann A (1905) Biologie der Trichopteren-Puppe. Zoologiesches Jahrbuch (Abteilung für Systematiek) 22: 489-574.

- Thomson RE (2012) Descriptions of New Species of Leucotrichiinae (Trichoptera: Hydroptilidae) from Brazil. Hindawi Publishing Corporation Psyche 2012: 1-7.

- Thomson RE, Holzenthal RW (2010) New Neotropical species of the genus Austrotinodes Schmid (Trichoptera: Ecnomidae). Zootaxa 2437: 38-50.

- Thomsom RE, Holzenthal RW (2012) New species and records of Hydroptilidae (Trichoptera) from Venezuela. Zookeys 185: 19. DOI: 10.3897/zookeys.185.2909

- $\quad$ Ulmer G (1905a) Neue und wenig bekannte aussereuropäische Trichopteren, hauptsächlich aus dem Wiener Museum. Annalen des kaiserlich-königlich naturhistorichen Hofmuseums 20: 59-98.

- $\quad$ Ulmer G (1905b) Zur Kenntniss aussereuropäischer Trichopteren. Stettiner Entomologische Zeitung 66 (1): 1-119.

- $\quad$ Ulmer G (1906) Neuer beitrag zur kenntnis aussereuropäischer Trichopteren. Notes from the Leyden Museum 28: 1-116.

- $\quad$ Ulmer G (1907a) Trichoptera. In: Wytsman P (Ed.) Genera Insectorum. 60. 1-259 pp.

- $\quad$ Ulmer G (1907b) Neue Trichopteren. Notes from the Leyden Museum 29: 1-53.

- Ulmer G (1907c) Monographie der Macronematinae. Collections Zoologiques du Baron Edm. de Selys Longchamps. 6. Hayez, Bruxelles, 1-121 pp.

- $\quad$ Ulmer G (1909) Einige neue exotische Trichopteren. Notes from the Leyden Museum 31: 125-142.

- Vallot JN (1855) Sur deux fourreaux hélicoïdes façonnés par les larves d'insectes. Mémoires de l'Académie des sciences, arts et belles-lettres de Dijon

- Vos RA, Biserkov JV, Balech B, Beard N, Blissett M, Brenninkmeijer C, Dooren TV, Eades D, Gosline G, Groom QJ, Hamman TD, Hettling H, Hoehndorf R, Holleman A, Hovenkamp P, Kelbert P, King D, Kirkup D, Lammers Y, DeMeulemeester T, Mietchen D, Miller JA, Mounce R, Nicolson N, Page R, Pawlik A, Pereira S, Penev L, Richards K, Sautter G, Shorthouse DP, Tähtinen M, Weiland C, Willians AR, Sierra S (2014)

Enriched biodiversity data as a resource and service. Biodiversity Data Journal 2: 1-15. DOI: 10.3897/BDJ.2.e1125

- Walker F (1852) Cataloque of the Specimens of Neuropterous Insects in the Collection of the British Museum, Part I: Phryganides-Perlides. British Museum, London, 192 pp. 
- Walker F (1860) Characters of undescribed Neuroptera in the collection of W. W. Saunders Esq. F. R. S. etc. Transactions of the Entomological Society of London 2 (5): 176-199.

- Wasmund AM, Holzenthal RW (2007) A revision of the Neotropical caddisfly genus Rhyacopsyche, with the description of 13 new species (Trichoptera: Hydroptilidae). 1634: 1-59.

- Yamamoto T (1966) Five new species of the caddisfly genus Polycentropus from South America (Trichoptera: Polycentropodidae). Canadian Entomologist 98: 908-912. DOI: 10.4039/Ent98908-9

- Yamamoto T (1967) New species of the caddisfly genus Polycentropus from Central America (Trichoptera: Polycentropodidae). Kansas Entomological Society 40 (2): 127-132.

\section{Supplementary materials}

\section{Suppl. material 1: Figure 1 source}

Authors: Paprocki, H., França, D.

Data type: spreadsheet, chart

Filename: Figure 1.xlsx - Download file (14.84 kb)

\section{Suppl. material 2: Figure 2 source}

Authors: Paprocki, H., França, D.

Data type: spreadsheet, chart

Filename: Figure 2.xlsx - Download file (12.04 kb) 